\title{
Rapeseed or linseed in grass-based diets: Effects on conjugated linoleic and conjugated linolenic acid isomers in milk fat from Holstein cows over 2 consecutive lactations
}

\author{
S. Lerch, ${ }^{*}$ K. J. Shingfield,† A. Ferlay, ${ }^{*}$ A. Vanhatalo, $\neq$ and Y. Chilliard ${ }^{* 1}$ \\ *INRA, UR1213 Herbivores, F-63122 Saint-Genès Champanelle, France \\ †MTT Agrifood Research Finland, Animal Production Research, FI-31600 Jokioinen, Finland \\ fUniversity of Helsinki, Department of Agricultural Sciences, PO Box 28, FI-00014 University of Helsinki, Finland
}

\begin{abstract}
Changes in the distribution of conjugated linoleic (CLA) and conjugated linolenic (CLnA) acid isomers in milk from Holstein cows in response to 4 different oilseed supplements rich in either cis-9 18:1 or 18:3n-3 were determined over 2 consecutive lactations in 58 and 35 cows during the first and second years, respectively. For the first 5 wk of the first lactation, all cows were fed the same diet. Thereafter, cows received 1 of 5 treatments for 2 consecutive lactations, including the prepartum period. Treatments comprised the basal diet with no additional lipid, or supplements of extruded linseeds (EL), extruded rapeseeds (ER), cold-pressed fat-rich rapeseed meal, or whole unprocessed rapeseeds to provide 2.5 to $3.0 \%$ of additional oil in diet dry matter. During indoor periods, cows were housed and received a mixture (3:1, wt/wt) of grass silage and hay, whereas cows were at pasture during outdoor periods. Over the entire study, EL resulted in the enrichment of $\Delta 11,13$ CLA, $\Delta 12,14$ CLA, trans-9,trans-11 CLA, trans-13,trans-15 CLA, $\Delta 9,11,15$ CLnA, and cis9,trans-11,trans-13 CLnA (identified for the first time in bovine milk fat) in milk fat, whereas ER and coldpressed fat-rich rapeseed meal in particular, increased milk fat trans-7,cis-9 CLA concentration. With the exception of the first indoor period, whole unprocessed rapeseeds decreased cis-9,trans-11 CLA, trans-9, cis-11 CLA, and trans-10,trans-12 CLA abundance. During the second indoor period, EL increased milk trans9,cis-11 CLA and trans-10,cis-12 CLA concentrations, but the increases in cis-9,trans-11 CLA, cis-12,trans-14 CLA, trans-11,cis-13 CLA, and cis-9,trans-11,cis-15 CLnA concentrations to EL and ER were lower for the second than first indoor period. In contrast to the indoor periods, EL and ER decreased milk cis-9,trans-11 CLA, trans-9,cis-11 CLA, and trans-10,cis-12 CLA concentrations at pasture. The extent of changes in the
\end{abstract}

Received April 23, 2012.

Accepted July 29, 2012.

${ }^{1}$ Corresponding author: yves.chilliard@clermont.inra.fr relative distribution and abundance of CLA and CLnA isomers in milk fat were related to the nature (rapeseed or linseed) and form of oilseed (extruded, cold-pressed fat-rich meal or whole unprocessed) supplement and their interactions with the composition of the basal diet (conserved grass or pasture and dietary starch content). Furthermore, milk fat CLA and CLnA responses to treatments were repeatable between both outdoor periods. Variations in milk fat content and yield measured during the entire study were significantly and inversely associated with milk trans-10 18:1, trans-10,cis-12 CLA, and in particular, trans-9, cis-11 CLA concentrations.

Key words: milk conjugated linoleic acid, milk conjugated linolenic acid, processed rapeseed, extruded linseed

\section{INTRODUCTION}

The terms conjugated linoleic acid (CLA) and conjugated linolenic acid (CLnA) refer to positional and geometric isomers of linoleic acid (cis-9, cis-12 18:2) and -linolenic acid (cis-9,cis-12,cis-15 18:3), respectively, in which 2 (CLA and conjugated diene CLnA) or 3 (fully conjugated CLnA) double bonds have a conjugated arrangement instead of methylene interruption. Ruminant fat is relatively abundant in CLA and conjugated diene CLnA (Collomb et al., 2006; Plourde et al., 2007; Gómez-Cortés et al., 2009), whereas fully conjugated CLnA are generally encountered in certain plant seeds including pomegranate, tung, bitter gourd, catalpa, and pot marigold (Hopkins and Chisholm, 1968).

Studies in animal models and human cell lines have provided evidence that certain isomers of CLA, including cis-9,trans-11 CLA, exhibit potent antiinflammatory, immunomodulatory, antiobesity, and anticarcinogenic activities and improve biomarkers of cardiovascular health (Wahle et al., 2004; Shingfield et al., 2008). Further research has shown that isomers of CLnA have similar biological effects (Koba et al., 2007; Hennessy et al., 2011), suggesting that dietary supplements of CLnA may have a positive role in regulating blood 
glucose and body composition in humans (BassaganyaRiera et al., 2011).

Based on the potential benefits on human health, there has been substantial interest in the potential to increase CLA concentration of ruminant milk. Available data also suggest that increasing milk fat CLnA concentrations may be beneficial. Numerous experiments have shown that dietary oilseed supplements induce changes in milk CLA isomer distributions according to the nature and form of oilseed supplement and composition of the basal diet (Palmquist et al., 2005; Collomb et al., 2006; Chilliard et al., 2007). However, most studies have characterized changes in milk fat CLA concentrations following a relatively short time on experimental diets, even though milk fat composition responses to dietary oilseed supplements are time dependent and may require several weeks to stabilize (Roy et al., 2006; Chilliard et al., 2007). Relatively few studies have reported the concentrations of CLnA in bovine milk fat, with observations limited to the effects of dietary raw or extruded linseeds; rapeseed, sunflower, or linseed oils; and camelina oil or expeller supplements (Akraim et al., 2007; Rego et al., 2009; Halmemies-BeauchetFilleau et al., 2011).

Interactions between supplements of sunflower oil (rich in 18:2n-6) and dietary starch contents have been shown to influence the distribution of CLA isomers in bovine milk (Roy et al., 2006). Recently, we examined the changes in milk FA composition due to extruded linseeds or different forms of rapeseed supplements in cows fed grass-based diets over 2 consecutive lactations (Lerch et al., 2012b), which revealed important interactions between oilseed supplementation and dietary starch content on the relative abundance of milk trans 18:1 and trans nonconjugated 18:2 isomers. However, no single study has examined short- or long-term effects of rapeseed (rich in cis-9 18:1) or linseed (rich in 18:3n-3) supplements in diets varying in starch content on the distribution of CLA and CLnA isomers in bovine milk fat.

The objective of this study was to characterize the relative abundance of CLA and partial CLnA isomers in milk fat of cows fed grass-based diets supplemented with extruded oilseeds of different nature (linseed or rapeseed) or form of rapeseed (extruded seeds, coldpressed fat-rich meal, or whole unprocessed seeds) over 2 consecutive lactations.

\section{MATERIALS AND METHODS}

\section{Animals and Diets}

Details on the experimental design have been presented previously (Lerch et al., 2012a). In brief, the experiment was conducted over 2 consecutive lactations, including an indoor overwintering period (grass silage- and grass hay-based diet, first half of lactation) and an outdoor period in the summer (grazing pasture-based diet, second half of lactation) per lactation. Fifty-eight and 35 (among the first 58) Holstein cows were used during the first and the second year of experimentation, respectively. During indoor periods, cows received a mixture of grass silage and grass hay of $3: 1$ and $4: 1$ on DM basis for the first and second year, respectively. During the outdoor period, cows were gradually turned out to pasture over a period of $1 \mathrm{wk}$, and then grazed for $20 \mathrm{~h} / \mathrm{d}$ from May 5, 2008, and May 4, 2009, to October 27, 2008, and November 3, 2009, during the first and the second year, respectively. In the first year, all cows received a diet without oilseed supplement from calving until the end of wk 5 of lactation. At the end of this period, cows were assigned to 1 of 5 groups according to calving date, parity, milk yield, and milk fat and protein content. Thereafter, experimental concentrates were introduced in the diet from wk 6 of lactation until the end of the second year of the study. A control concentrate comprised pelleted wheat and solvent-extracted rapeseed meal (control, CTL), which was partially substituted by extruded linseeds [EL: extruded blend of linseeds (70:30, wt/wt) and wheat; the mixture was extruded for $15 \mathrm{~s}$ at $100^{\circ} \mathrm{C}$ and then dried for $20 \mathrm{~min}$ at 125 to $145^{\circ} \mathrm{C}$; $\mathrm{INZO}^{\circ}$, Argentan, France], extruded rapeseeds [ER: extruded blend (59:29:12, wt/wt/wt) of rapeseeds, wheat, and wheat bran; the mixture was extruded for $15 \mathrm{~s}$ at $100^{\circ} \mathrm{C}$ and then dried for $20 \mathrm{~min}$ at 125 to $145^{\circ} \mathrm{C}$; INZO ${ }^{\circ}$, cold-pressed fat-rich rapeseed meal (FRM: byproduct of rapeseed oil extraction by cold pressure; Dock Moulin SA, Marneffe, Belgium), or unprocessed, full-fat, uncrushed, whole rapeseeds $\left(\mathbf{W R} ; \mathrm{INZO}^{\circ}\right)$. From wk 6 of lactation to the end of the indoor periods, the amount of concentrate offered was adjusted weekly to maintain a 70:30 and 60:40 forage-to-concentrate ratio during the first and the second year, respectively. During both outdoor periods, each cow received $4.5 \mathrm{~kg}$ of concentrate DM/d. Cows also received $4.5 \mathrm{~kg}$ of concentrate $\mathrm{DM} / \mathrm{d}$ of the same composition as fed during the first outdoor period in the dry period.

Oilseed supplements were offered to provide an estimated 3.0 and $2.5 \%$ of oil in diet DM during the indoor and outdoor periods, respectively. Chemical composition of the concentrate mixtures used throughout the experiment was dependent on the year of study and the indoor and outdoor feeding period (see Lerch et al., 2012a). Cows were milked at 0630 and $1600 \mathrm{~h}$ in a milking parlor and were housed in a freestall barn during indoor periods. 


\section{Sampling, Measurements, and Chemical Analyses}

Individual forage and concentrate intakes were recorded and starch, ether extract contents, and FA composition of feedstuffs were determined at periodic intervals as outlined elsewhere (Lerch et al., 2012a,b). For the indoor periods, dietary 18:2n-6 and 18:3n-3 contents were estimated based on estimates of total FA in ether extract (Morand-Fehr and Tran, 2001; Sauvant et al., 2004). For the outdoor periods, diet 18:2n-6, 18:3n3 , and starch content were predicted from estimates of pasture intake (Faverdin et al., 2007).

Milk. Daily milk yield was recorded for all cows, whereas samples of milk were collected on 10 occasions throughout the experiment [i.e., twice during the first indoor period $(72 \pm 17.0$ and $141 \pm 23.1$ DIM; mean $\pm \mathrm{SD}$ ), 3 times during the first outdoor period (197 \pm 23.1, $232 \pm 23.1$, and $288 \pm 23.1$ DIM; mean \pm SD), twice during the second indoor period $(84 \pm 13.3$ and $129 \pm 35.0$ DIM; mean \pm SD), and 3 times during the second outdoor period $(171 \pm 35.0,199 \pm 35.0$, and $262 \pm 35.0$ DIM; mean $\pm \mathrm{SD})]$. Milk samples $(30 \mathrm{~mL})$ were collected over 4 consecutive milkings, preserved in tubes containing bronopol B-2 (Trillaud Surgères, France), stored at $4^{\circ} \mathrm{C}$, and analyzed for fat content (Galilait, Theix, France) using mid-infrared spectrometry (AOAC International, 1997; Milkoscan 4000; Foss Electric A/S; Hillerød, Denmark). At the same time, subsamples $(3 \mathrm{~mL})$ were collected over 2 consecutive milkings and stored at $-20^{\circ} \mathrm{C}$ before lyophilization and milk FA determination. For each cow, lyophilized milk samples of morning and evening milking were composited (60 and $40 \mathrm{mg}$ of lyophilized milk for morning and evening milkings, respectively) before direct methylation.

\section{Lipid Analysis.}

Preparation of FA Derivatives and Analysis. Fatty acids in 100-mg lyophilized milk samples were converted to FA methyl esters (FAME) by direct methylation (Lerch et al., 2012b). The FAME were analyzed using GC (Trace-GC 2000 Series gas chromatograph; Thermo Finnigan, Les Ulis, France) equipped with a flame ionization detector and a 100-m fused silica capillary column (i.d. $0.25 \mathrm{~mm}$ ) coated with a $0.2-\mu \mathrm{m}$ film of cyanopropyl polysiloxane (CP-Sil88; Chrompack, Middelburg, the Netherlands). The total milk FAME distribution in a $0.6-\mu \mathrm{L}$ sample at a split ratio of 1:50 was determined using an oven temperature program (Lerch et al., 2012b). Peaks were routinely identified by comparison of the retention time with commercial authentic standards containing a mixture of FAME (GLC \#463, Nu-Chek Prep, Elysian, MN; Supelco \#37, Supelco Inc., Bellefonte, PA), methyl esters of geometric isomers of $\Delta 9,12$ 18:2 (L8404; Sigma,
Steinheim, Germany), positional and geometric isomers of CLA (O5632; Sigma), and cis-9,trans-11,trans-13 CLnA (\#10-1803-23-7; Larodan Fine Chemicals AB, Malmö, Sweden).

Methyl esters not available as authentic standards were identified according to the electron impact ionization spectra of FAME and 4,4-dimethyloxazoline (DMOX) derivatives obtained by GC-MS. Preparation of DMOX derivatives, parameters used for GC-MS analysis, and interpretation of mass spectra were in accordance with earlier reports (Halmemies-BeauchetFilleau et al., 2011), with the exception that the temperature program applied during GC-MS analysis was identical to that used for the analysis of the FAME by GC.

Treatment effects on milk FA composition $(\mathrm{n}=465)$ have been reported previously (Lerch et al., 2012b). Subsequent investigations enabled 3 isomers of CLnA isomers to be identified in all 465 milk samples. Due to the large number of samples generated, a smaller subset of 186 samples of milk collected at the end of each feeding period (i.e., $141 \pm 23.1$ DIM during first indoor, $288 \pm 23.1$ DIM during first outdoor, $129 \pm 35.0$ DIM during second indoor, and $262 \pm 35.0$ DIM during second outdoor periods; mean $\pm \mathrm{SD}$ ) were selected for detailed analysis of CLA composition. The distribution of CLA isomers in milk fat FAME was determined using an HPLC system (model 1080; Hewlett-Packard Co., Wilmington, DE) equipped with 4 silver-impregnated silica columns (ChromSpher 5 Lipids, $250 \times 4.6 \mathrm{~mm}$, $5-\mu \mathrm{m}$ particle size; Varian Ltd., Walton-on-Thames, UK) coupled in series. Methyl esters of CLA were separated under isocratic conditions at $22^{\circ} \mathrm{C}$ using $0.1 \%$ ( $\mathrm{vol} / \mathrm{vol}$ ) acetonitrile in heptane at a flow rate of $1 \mathrm{~mL} /$ min and monitoring effluent at 233 and $210 \mathrm{~nm}$ (Shingfield et al., 2003). Identification of CLA isomers was performed based on retention time comparisons with a mixture of authentic standards (O5632; Sigma), and co-injection with methyl esters prepared from a range of materials enriched in specific CLA isomers (Sæbø et al., 2005; Shingfield et al., 2005). Concentrations of CLA isomers were calculated from the proportionate peak area responses determined by HPLC and the sum of concentrations of trans-7,cis-9 CLA, trans-8, cis-10 CLA, and cis-9,trans-11 CLA weight percentage determined by GC analysis.

Identification of $\alpha$-Eleostearic Acid (cis9,trans-11,trans-13 CLnA) in Milk Fat. A partial gas chromatogram indicating the separation and elution order of CLnA isomers is shown in Figure 1. Two geometrical isomers of $\Delta 9,11,15$ CLnA eluted with a mean retention time of 54.4 and 57.5 min (peaks no. 4 and no. 6, respectively, in Figure 1). An additional CLnA isomer eluted between 20:5n-3 and 24:0 with a 
retention time of 68.5 min (peak no. 16 in Figure 1). For all 3 isomers, GC-MS analysis of FAME revealed a molecular ion at $m / z 292$, confirming the occurrence of 18-carbon methyl ester containing 3 double bonds. An 18:3 structure was verified based on a molecular ion at $m / z 331$ during GC-MS analysis of the corresponding DMOX derivative (Supplementary Figure 1, available online at http://www.journalofdairyscience.org/).

Mass spectra of the DMOX derivatives of peaks no. 4 (cis-9,trans-11,trans-15 CLnA) and no. 6 (cis-9,trans11,cis-15 CLnA) were similar and, therefore, only the mass spectrum of peak no. 6 is reported (Supplementary Figure 1A, available online at http://www.journalofdairyscience.org/). Double bonds at $\Delta 9, \Delta 11$, and $\Delta 15$, were located based on 12-amu gaps between ion fragments at $\mathrm{m} / z 196$ and 208, 222 and 234, and 276 and 288. Interpretation of a $\Delta 9,11,15$ CLnA structure was confirmed based on comparison with published data (Plourde et al., 2007; Gómez-Cortés et al., 2009). The configuration of the cis,trans,trans and cis,trans, cis double bonds for peaks no. 4 and no. 6, respectively, was deduced from relative retention times during GC analysis and comparison with reports in the literature (Gómez-Cortés et al., 2009).

The mass spectrum of the DMOX derivative of peak no. 16 (cis-9,trans-11,trans-13 CLnA) (Supplementary Figure 1B, available online at http://www.journalofdairyscience.org/) revealed ion fragments at $\mathrm{m} / z 196$ and 208, 222 and 234, and 248 and 260, locating the occurrence of double bonds at $\Delta 9, \Delta 11$, and $\Delta 13$, respectively. A $\Delta 9,11,13$ 18:3 structure was further supported based on the presence of 3 intense ions at $m / z 182,288$, and 302 arising from allylic cleavages to the proximal (containing $\mathrm{n}-2$ carbons, $m / z$ 182) and distal double bonds (containing $\mathrm{m}+2$ carbons, $m / z 288$, where $\mathrm{n}$ and $\mathrm{m}$ denote the first carbon of the proximal and distal double bond, respectively). The mass spectrum of the $\Delta 9,11,13$ CLnA DMOX derivative isolated in milk fat was found to be identical to earlier reports in the literature (Spitzer et al., 1994). A cis,trans,trans configuration of double bonds was confirmed by coinjection with an authentic standard.

\section{Calculations and Statistical Analyses}

Diet 18:2n-6, 18:3n-3 and starch contents, milk yield, milk fat content and yield, and milk FA composition data were analyzed using the MIXED procedure of SAS (SAS Institute Inc., Cary, NC; 2003). Measurements for the indoor (Table 1 and Figure 2 and 3 indoor period panels) and outdoor (Table 2 and Figure 2 and 3 outdoor period panels) periods were analyzed separately. The model for repeated measures included treatment, year, and parity at the first year of experimentation within year, and treatment by year interaction as fixed effects, and cow as a random effect using a compound

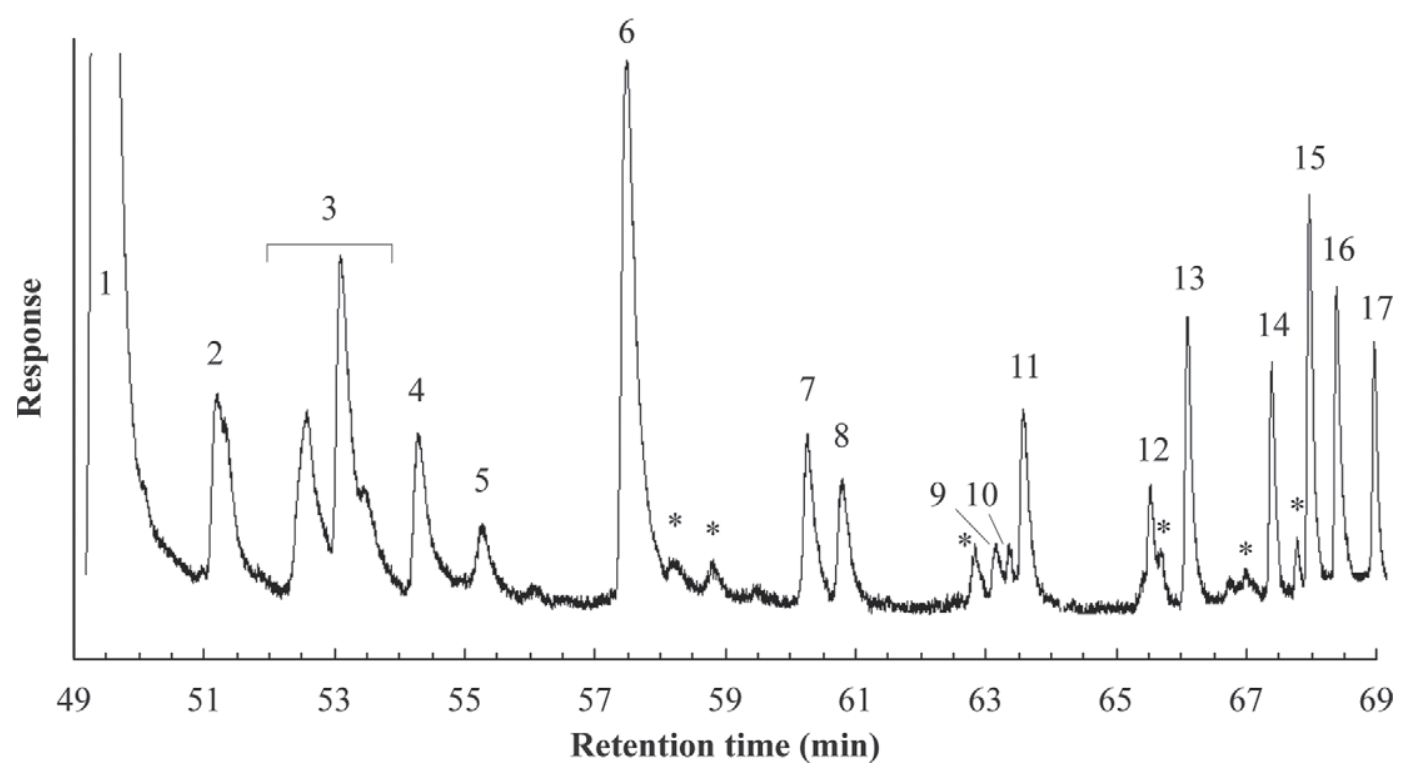

Figure 1. Partial gas chromatogram indicating the elution of conjugated linoleic (CLA) and conjugated linolenic acid (CLnA) isomers obtained using a temperature gradient for FA methyl esters prepared from a sample of milk fat from 1 cow fed extruded linseeds at pasture. Identification was verified based on electron impact ionization spectra recorded during GC-MS analysis of FA methyl esters and corresponding 4,4-dimethyloxazoline derivatives. Peak identification: $1=$ unresolved cis-9,trans-11 CLA, trans-7,cis-9 CLA, and trans-8,cis-10 CLA; $2=$ unresolved 21:0, cis-9,cis-11 CLA, and trans-11,cis-13 CLA; $3=$ unresolved trans-12,trans-14 CLA, trans-11,trans-13 CLA, trans-10,trans-12 CLA, and trans-9,trans-11 CLA; $4=$ cis-9,trans-11,trans-15 CLnA; $5=$ cis-11,cis-14 20:2; $6=$ cis-9,trans-11,cis-15 CLnA; $7=22: 0 ; 8=$ cis8,cis-11,cis-14 20:3; $9=$ cis-11,cis-14,cis-17 20:3; $10=$ cis-13 22:1; $11=$ cis-5,cis-8,cis-11,cis-14 20:4; $12=23: 0 ; 13=$ cis-8,cis-11,cis-14,cis-17 20:4; $14=$ cis-14 23:1; $15=$ cis-5,cis-8,cis-11,cis-14,cis-17 20:5; $16=$ cis-9,trans-11,trans-13 CLnA; $17=24: 0 ;{ }^{*}=$ unidentified. 
symmetry covariance structure. The statistical model used to analyze milk CLnA concentration data reported in Figure 3 also included week within year and treatment by week within year as fixed effects, and a spatial power covariance structure. For all statistical models, if the treatment by year or treatment by week within-year interactions were significant, the SLICE option was used to compare treatment differences at each sampling time. Values reported in Tables 1 and 2 and in Figures 2 and 3 are least squares means and associated standard error of the mean derived using the within period (indoor or outdoor) statistical models. Treatment differences were determined based on $t$-tests and declared significant at $P \leq 0.05$. Trends toward significance were considered at $0.05<P \leq 0.10$.

To test for the significance of interactions between treatment and period (indoor vs. outdoor), data from the first year and the second year of the experiment were analyzed separately. The model for repeated measures included treatment, period (indoor vs. outdoor), parity at the first year of experimentation, and treatment by period interaction (plus week within year and treatment by week within year for CLnA data) as the fixed effects, and cow as a random effect. Least squares means and standard error of the mean corresponding to the within-year models (yr 1 or 2) are not shown in the tables or figures, but the $P$-values of relevant comparisons arising from this analysis are reported in the text, as denoted by data not shown.

Milk fat concentrations of nonconjugated FA (Lerch et al., 2012b) along with current measurements of CLA and CLnA isomer abundance were used to explore the relationships between milk conjugated FA and nonconjugated 18-carbon FA using the CORR procedure of SAS. Data for individual cows were used to derive correlations between CLA and CLnA isomers, and 18-carbon FA ( $\mathrm{n}=186$ for CLA and $\mathrm{n}=465$ for CLnA). Pairwise correlations were examined using the entire set of data $(\mathrm{n}=465)$ or within-period subsets $(\mathrm{n}=186$ and 279 for the indoor and outdoor periods, respectively). Furthermore, the relation between concentrations of CLA and CLnA isomers containing a cis-9 double bond with the relative abundance of corresponding putative 18:1 and 18:2 precursors were examined by regression analysis using the GLM procedure of SAS.

Given that feeding period (linked to stage of lactation) and parity may influence milk fat content and yield, correlations between milk fat content and yield, CLA, CLnA, and 18-carbon FA concentrations were derived based on the difference between each treatment least squares means and the average of the 5 treatment least squares means for a given period ( 2 indoor and 2 outdoor periods), resulting in 20 comparisons.
To further explore the association between CLA and CLnA isomers, and between conjugated and other FA, measurements of milk fat composition of all cows fed the EL treatment $(\mathrm{n}=40)$ were analyzed by principal components analysis (PCA) using Statistica version 6.1 software (StatSoft Inc., Maisons-Alfort, France; 2004). A preliminary analysis was undertaken using data that included all measured 18-carbon FA isomer concentrations. Thereafter, the abundance of FA with a similar degree of unsaturation and double-bond configuration, exhibiting much the same pattern defined as coordinates that were closely aligned according to all 3 first principal components, were paired together (i.e., cis-12 18:1 + cis-13 18:1; trans-4 18:1 + trans-5 18:1; trans,-6,-7,-8 18:1 + trans-9 18:1; trans-13,-14 18:1 + trans-16 18:1, and cis-9,trans-13 18:2 + cis-9,trans-14 18:2) to decrease the number of active variables to 37 . Starch intake and milk fat content were included into the PCA as passive variables.

\section{RESULTS}

\section{Diet Fat and Starch Contents, Milk Yield, and Milk Composition}

Irrespective of study year and feeding period, diet 18:2n-6 content was higher for oilseed treatments than for the CTL, especially during the second indoor period. Diet 18:3n-3 content was higher for oilseed treatments relative to the CTL, for EL, and during indoor periods in particular (Figure $2 \mathrm{~A} ; P<0.001$ ). In contrast, diet starch content was consistently higher for the CTL than for diets containing oilseeds (Figure 2B; $P<0.001$ ). Diet starch content was also higher during the second indoor period than for all other periods. Furthermore, for the second indoor period, differences in starch content between the CTL and the oilseed treatments were lower than at other times during the experiment (Figure 2B; $P<0.001$ ).

Milk yield was not affected $(P>0.10)$ by treatment (Tables 1 and 2). During the second indoor period, milk fat content and yield were higher $(P<0.01)$ for FRM and WR compared with other treatments (Table 1). Milk fat content was higher $(P=0.01)$ for WR than for other treatments during both outdoor periods, whereas the fat content of milk was higher $(P=0.01)$ for EL relative to the CTL during the second outdoor period (Table 2). However, treatment had no effect $(P=0.41)$ on milk fat secretion during both outdoor periods.

\section{Milk CLA and CLnA Composition}

Effects of Oilseed Nature (EL vs. ER) During yr 1. During the first indoor period, milk total CLA 
Table 1. Effects of oilseed supplements on milk yield, milk fat content and secretion, and milk conjugated linoleic acid (CLA) and conjugated linolenic acid (CLnA) composition during consecutive indoor periods in lactating cows

\begin{tabular}{|c|c|c|c|c|c|c|c|c|c|c|c|c|c|c|}
\hline \multirow[b]{3}{*}{ Item } & \multicolumn{5}{|c|}{ yr $1(2008, \mathrm{n}=58)^{1}$} & \multicolumn{5}{|c|}{ yr $2(2009, \mathrm{n}=35)^{1}$} & \multirow[b]{3}{*}{ SEM } & \multicolumn{3}{|c|}{$P$-value } \\
\hline & \multicolumn{5}{|c|}{ Treatment $^{2}$} & \multicolumn{5}{|c|}{ Treatment $^{2}$} & & \multirow[b]{2}{*}{ Treatment } & \multirow[b]{2}{*}{ Year } & \multirow{2}{*}{$\begin{array}{l}\text { Treatment } \\
\quad \times \text { year }\end{array}$} \\
\hline & CTL & EL & $\mathrm{ER}$ & FRM & WR & CTL & EL & ER & FRM & WR & & & & \\
\hline Milk yield $(\mathrm{kg} / \mathrm{d})$ & 26.1 & 25.0 & 26.9 & 26.1 & 25.3 & 29.3 & 29.3 & 33.9 & 32.1 & 28.4 & 1.05 & 0.12 & $<0.001$ & 0.13 \\
\hline Content (\%) & 3.56 & 3.49 & 3.40 & 3.42 & 3.62 & $3.00^{\mathrm{b}}$ & $2.94^{\mathrm{b}}$ & $2.82^{\mathrm{b}}$ & $3.58^{\mathrm{a}}$ & $3.72^{\mathrm{a}}$ & 0.131 & 0.02 & $<0.001$ & $<0.001$ \\
\hline Yield $(\mathrm{g} / \mathrm{d})$ & 930 & 869 & 913 & 894 & 913 & $876^{\mathrm{b}}$ & $871^{\mathrm{b}}$ & $953^{\mathrm{b}}$ & $1,144^{\mathrm{a}}$ & $1,046^{\mathrm{a}}$ & 43.9 & 0.11 & $<0.01$ & $<0.01$ \\
\hline \multicolumn{15}{|c|}{ Concentration $(\mathrm{mg} / 100 \mathrm{~g}$ of FA) } \\
\hline cis-9,trans-11 CLA & $586^{\mathrm{c}}$ & $891^{\mathrm{a}}$ & $727^{\mathrm{b}}$ & $746^{\mathrm{b}}$ & $559^{\mathrm{c}}$ & $688^{\mathrm{b}}$ & $910^{\mathrm{a}}$ & $760^{\mathrm{b}}$ & $895^{\mathrm{a}}$ & $484^{\mathrm{c}}$ & 31.3 & $<0.001$ & 0.08 & 0.02 \\
\hline cis-11,trans-13 CLA & $1.23^{\mathrm{b}}$ & $2.50^{\mathrm{a}}$ & $1.63^{\mathrm{b}}$ & $1.59^{\mathrm{b}}$ & $1.72^{\mathrm{b}}$ & $1.79^{\mathrm{b}}$ & $4.54^{\mathrm{a}}$ & $2.27^{\mathrm{b}}$ & $1.85^{\mathrm{b}}$ & $1.87^{\mathrm{b}}$ & 0.268 & $<0.001$ & $<0.01$ & 0.06 \\
\hline cis-12,trans-14 CLA & $0.85^{\mathrm{b}}$ & $3.90^{\mathrm{a}}$ & $1.09^{\mathrm{b}}$ & $1.10^{\mathrm{b}}$ & $1.11^{\mathrm{b}}$ & $1.12^{\mathrm{bc}}$ & $2.16^{\mathrm{a}}$ & $0.57^{\mathrm{c}}$ & $1.45^{\mathrm{b}}$ & $0.73^{\mathrm{c}}$ & 0.159 & $<0.001$ & $<0.01$ & $<0.001$ \\
\hline trans-7,cis-9 CLA & $30.4^{\mathrm{d}}$ & $58.5^{\mathrm{c}}$ & $78.4^{\mathrm{b}}$ & $103.1^{\mathrm{a}}$ & $38.3^{\mathrm{d}}$ & $54.0^{\mathrm{c}}$ & $102.6^{\mathrm{b}}$ & $123.9^{\mathrm{a}}$ & $128.8^{\mathrm{a}}$ & $52.4^{\mathrm{c}}$ & 3.53 & $<0.001$ & $<0.001$ & $<0.001$ \\
\hline trans-9,cis-11 CLA & $\begin{array}{r}7.4 \\
7.9\end{array}$ & 10.7 & 9.4 & $\begin{array}{r}100.1 \\
9.9\end{array}$ & 7.6 & $15.0^{\mathrm{bc}}$ & $24.9^{\mathrm{a}}$ & $20.7^{\mathrm{ab}}$ & $13.0^{\mathrm{cd}}$ & $8.4^{\mathrm{d}}$ & $\begin{array}{l}0.00 \\
1.28\end{array}$ & $<0.001$ & $<0.001$ & $<0.001$ \\
\hline trans-10,cis-12 CLA & 4.03 & 2.28 & 3.65 & 2.64 & 2.58 & $11.02^{\mathrm{b}}$ & $17.26^{\mathrm{a}}$ & $13.61^{\mathrm{b}}$ & $6.46^{\mathrm{c}}$ & $4.40^{\mathrm{c}}$ & 0.792 & $<0.001$ & $<0.001$ & $<0.001$ \\
\hline trans-11.cis-13 CLA & $26.9^{\mathrm{c}}$ & $77.9^{\mathrm{a}}$ & $38.9^{\mathrm{b}}$ & $31.5^{\mathrm{c}}$ & $41.7^{\mathrm{b}}$ & $11.9^{\mathrm{b}}$ & $23.1^{\mathrm{a}}$ & $12.6^{\mathrm{b}}$ & $18.4^{\mathrm{ab}}$ & $13.5^{\mathrm{b}}$ & 2.29 & $<0.001$ & $<0.001$ & $<0.001$ \\
\hline trans-12,cis-14 CLA & $3.0^{\mathrm{b}}$ & $23.0^{\mathrm{a}}$ & $4.6^{\mathrm{b}}$ & $4.9^{\mathrm{b}}$ & $3.6^{\mathrm{b}}$ & $3.5^{\mathrm{b}}$ & $31.0^{\mathrm{a}}$ & $4.3^{\mathrm{b}}$ & $5.5^{\mathrm{b}}$ & $3.6^{\mathrm{b}}$ & 0.92 & $<0.001$ & $<0.01$ & $<0.001$ \\
\hline trans-7.trans-9 CLA & 1.69 & 1.73 & 2.66 & 2.60 & 2.02 & 2.59 & 2.16 & 2.92 & 2.28 & 2.62 & 0.214 & 0.11 & 0.05 & 0.29 \\
\hline trans-8,trans-10 CLA & 2.40 & 2.57 & 3.11 & 3.17 & 2.91 & $3.74^{\mathrm{a}}$ & $2.48^{\mathrm{b}}$ & $1.81^{\mathrm{b}}$ & $2.19^{\mathrm{b}}$ & $2.81^{\mathrm{ab}}$ & 0.271 & 0.52 & 0.34 & 0.01 \\
\hline trans-9,trans-11 CLA & $9.1^{\mathrm{b}}$ & $16.2^{\mathrm{a}}$ & $10.6^{\mathrm{b}}$ & $10.7^{\mathrm{b}}$ & $9.1^{\mathrm{b}}$ & $11.5^{\mathrm{b}}$ & $16.5^{\mathrm{a}}$ & $11.3^{\mathrm{b}}$ & $16.3^{\mathrm{a}}$ & $9.5^{\mathrm{b}}$ & 0.58 & $<0.001$ & $<0.001$ & $<0.001$ \\
\hline trans-10,trans-12 CLA & 3.43 & 3.60 & 3.20 & 3.99 & 2.60 & $6.75^{\mathrm{a}}$ & $5.80^{\mathrm{ab}}$ & $4.35^{\mathrm{bc}}$ & $6.03^{\mathrm{a}}$ & $3.70^{\mathrm{c}}$ & 0.369 & $<0.001$ & $<0.001$ & 0.09 \\
\hline trans-11,trans-13 CLA & $15.6^{\mathrm{c}}$ & $52.1^{\mathrm{a}}$ & $24.6^{\mathrm{b}}$ & $26.4^{\mathrm{b}}$ & $19.0^{\mathrm{c}}$ & $15.2^{\mathrm{c}}$ & $47.7^{\mathrm{a}}$ & $13.7^{\mathrm{c}}$ & $35.1^{\mathrm{b}}$ & $12.8^{\mathrm{c}}$ & 1.60 & $<0.001$ & $<0.01$ & $<0.001$ \\
\hline trans-12,trans-14 CLA & $7.7^{\mathrm{d}}$ & $40.4^{\mathrm{a}}$ & $12.8^{\mathrm{c}}$ & $\begin{array}{l}20.4 \\
12.8^{\mathrm{b}}\end{array}$ & $9.0^{\mathrm{cd}}$ & $\begin{array}{r}10.4^{\mathrm{d}} \\
7.4^{2}\end{array}$ & $39.0^{\mathrm{a}}$ & $\begin{aligned} 1.1 . \\
8.7^{\mathrm{c}}\end{aligned}$ & $15.5^{\mathrm{b}}$ & $7.2^{\mathrm{cd}}$ & $\begin{array}{l}1.00 \\
1.04\end{array}$ & $<0.001$ & 0.19 & 0.11 \\
\hline trans-13,trans-15 CLA & $0.66^{\mathrm{c}}$ & $3.19^{\mathrm{a}}$ & $1.28^{\mathrm{b}}$ & $1.44^{\mathrm{b}}$ & $0.83^{\mathrm{c}}$ & $0.74^{\mathrm{bc}}$ & $4.06^{\mathrm{a}}$ & $0.67^{\mathrm{bc}}$ & $1.17^{\mathrm{b}}$ & $0.61^{\mathrm{c}}$ & 0.109 & $<0.001$ & 0.82 & $<0.01$ \\
\hline$\Sigma \mathrm{CLA}^{3}$ & $708^{\mathrm{c}}$ & $1,200^{\mathrm{a}}$ & $930^{\mathrm{b}}$ & $971^{\mathrm{b}}$ & $708^{\mathrm{c}}$ & $843^{\mathrm{b}}$ & $1,244^{\mathrm{a}}$ & $973^{\mathrm{b}}$ & $1,161^{\mathrm{a}}$ & $612^{\mathrm{c}}$ & 37.8 & $<0.001$ & 0.02 & $<0.01$ \\
\hline $\begin{array}{l}\text { cis-9,trans-11,cis- } 15 \\
\text { CLnA }\end{array}$ & $61.4^{\mathrm{d}}$ & $298.4^{\mathrm{a}}$ & $127.4^{\mathrm{b}}$ & $91.7^{\mathrm{cd}}$ & $98.9^{\mathrm{bc}}$ & $34.5^{\mathrm{b}}$ & $96.3^{\mathrm{a}}$ & $28.2^{\mathrm{b}}$ & $57.4^{\mathrm{b}}$ & $47.9^{\mathrm{b}}$ & 11.15 & $<0.001$ & $<0.001$ & $<0.001$ \\
\hline $\begin{array}{l}\text { cis-9,trans-11,trans- } 13 \\
\text { CLnA }\end{array}$ & $9.9^{\mathrm{c}}$ & $27.6^{\mathrm{a}}$ & $23.4^{\mathrm{bc}}$ & $21.6^{\mathrm{b}}$ & $16.0^{\mathrm{c}}$ & $11.1^{\mathrm{c}}$ & $26.8^{\mathrm{a}}$ & $10.9^{\mathrm{bc}}$ & $16.0^{\mathrm{b}}$ & $7.8^{\mathrm{c}}$ & 2.33 & $<0.001$ & $<0.01$ & 0.18 \\
\hline $\begin{array}{l}\text { cis-9,trans-11,trans- } 15 \\
\text { CLnA }\end{array}$ & $33.2^{\mathrm{b}}$ & $62.5^{\mathrm{a}}$ & $40.8^{\mathrm{b}}$ & $36.6^{\mathrm{b}}$ & $32.0^{\mathrm{b}}$ & $19.9^{\mathrm{b}}$ & $51.5^{\mathrm{a}}$ & $20.7^{\mathrm{b}}$ & $25.8^{\mathrm{b}}$ & $14.9^{\mathrm{b}}$ & 4.42 & $<0.001$ & $<0.001$ & 0.91 \\
\hline$\sum \mathrm{CLnA}^{4}$ & $104.4^{\mathrm{d}}$ & $388.4^{\mathrm{a}}$ & $191.7^{\mathrm{b}}$ & $149.9^{\mathrm{c}}$ & $146.8^{\mathrm{c}}$ & $65.4^{\mathrm{b}}$ & $172.3^{\mathrm{a}}$ & $69.2^{\mathrm{b}}$ & $97.9^{\mathrm{b}}$ & $73.5^{\mathrm{b}}$ & 13.36 & $<0.001$ & $<0.001$ & $<0.001$ \\
\hline
\end{tabular}

${ }^{\mathrm{a}-\mathrm{d}}$ Within a row and year, means with different letters differ at $P<0.05$

${ }^{1}$ Measured on 1 occasion for each year of experiment during wk $20 \pm 3.3$ and $18 \pm 5.0$ (means \pm SD) of lactation for yr 1 and 2 , respectively

${ }^{2}$ Cows fed a grass silage- and hay-based diet containing no additional oilseed supplement (CTL; $\mathrm{n}=12$ and 7 during yr 1 and 2 , respectively), or 2.9 to $5.1 \%$ of oil in diet DM from extruded linseeds (EL; $\mathrm{n}=12$ and 8 during yr 1 and 2 , respectively), extruded rapeseeds (ER; $\mathrm{n}=12$ and 4 during yr 1 and 2 , respectively), cold-pressed fat-rich rapeseed meal (FRM; $\mathrm{n}=10$ and 8 during yr 1 and 2, respectively), or whole unprocessed rapeseeds (WR; $\mathrm{n}=12$ and 8 during yr 1 and 2 , respectively).

${ }^{3}$ Determined by GC and HPLC.

${ }^{4}$ Determined by GC. 

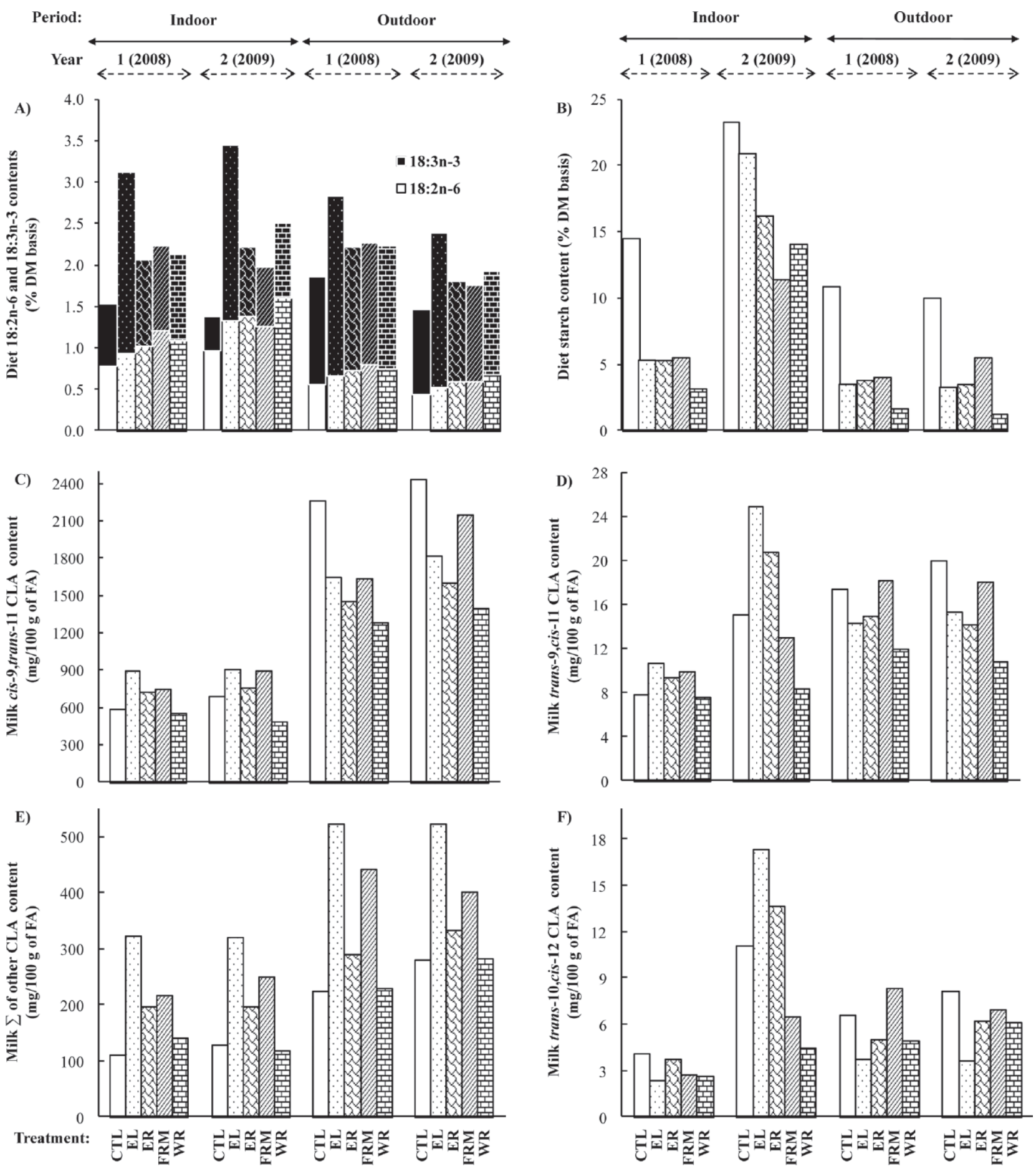

Figure 2. (A) Diet 18:2n-6 and 18:3n-3 contents, (B) diet starch content, (C) milk cis-9,trans-11 conjugated linoleic acid (CLA), (D) milk trans-9,cis-11 CLA, (E) milk sum of other milk CLA, and (F) trans-10,cis-12 CLA concentrations in cows housed indoors and fed grass silageand grass hay-based diets or at pasture, over 2 consecutive lactations $(\mathrm{n}=186)$. Cows received diets containing no additional lipid (control diet, CTL, $\mathrm{n}=12$ during the first year and $\mathrm{n}=7$ during the second year), or 2.5 to $5.1 \%$ of oil in diet DM from extruded linseeds (EL, $\mathrm{n}=12$ during the first year and $n=8$ during the second year), extruded rapeseeds (ER, $n=12$ during the first year and $n=4$ during the second year), coldpressed fat-rich rapeseed meal (FRM, $\mathrm{n}=10$ during the first year and $\mathrm{n}=8$ during the second year), or whole unprocessed rapeseeds (WR, $\mathrm{n}$ $=12$ during the first year and $n=8$ during the second year). Each point represents treatment LSM calculated within period (indoor or outdoor; refer to the Materials and Methods section). Effects of treatment and year were significant $(P<0.05)$, except for the influence of year on diet starch content, milk trans-9,cis-11 CLA, and milk trans-10,cis-12 CLA during outdoor periods, and the sum of other milk CLA isomers during both indoor and outdoor periods $(P>0.20)$. For all parameters, treatment by year was significant during indoor periods $(P<0.05)$, but was not significant $(P>0.20)$ during outdoor periods. 

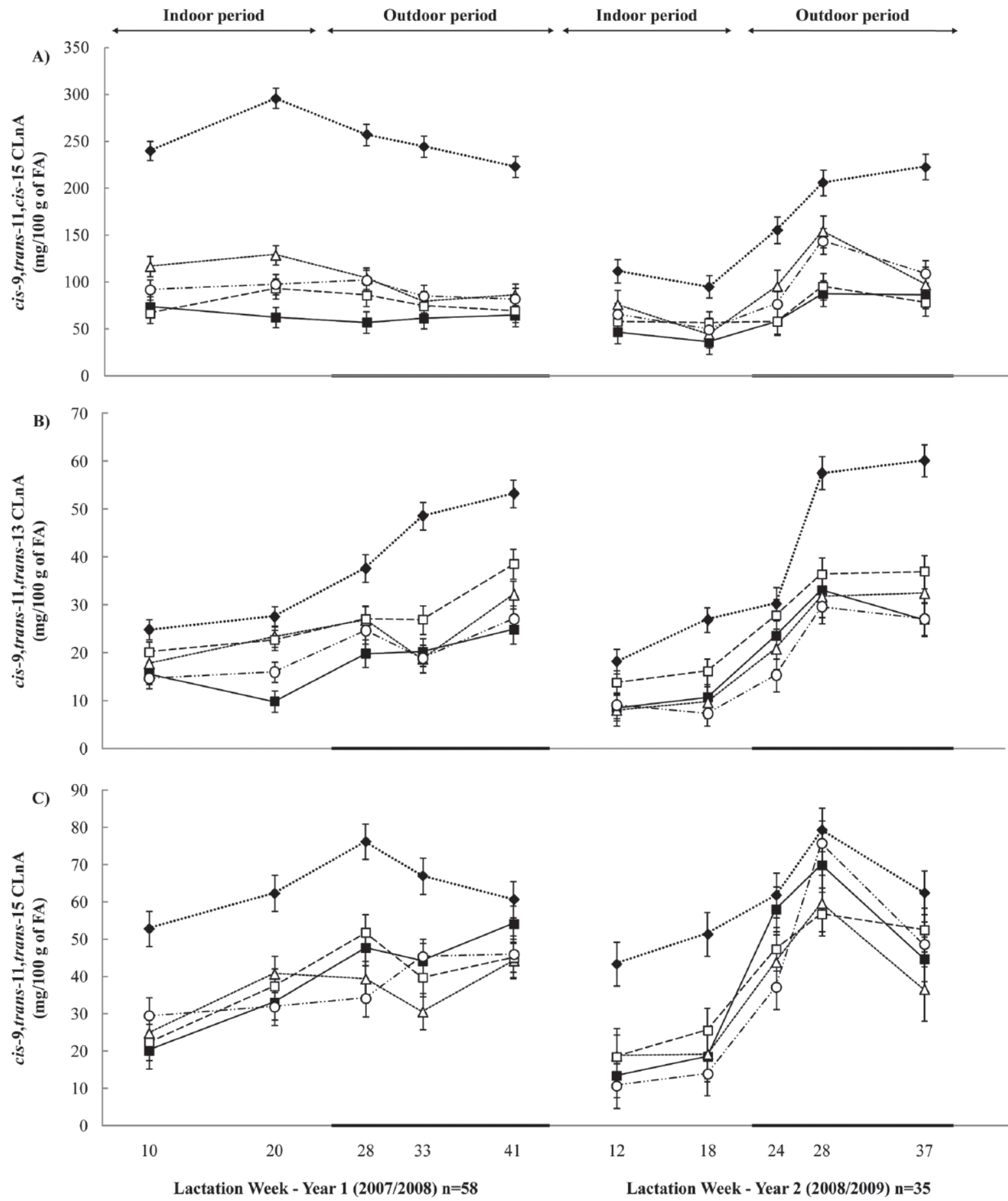

Figure 3. (A) Concentrations of cis-9,trans-11,cis-15 conjugated linolenic acid (CLnA), (B) cis-9,trans-11,trans-13 CLnA, and (C) cis9,trans-11,trans-15 CLnA in milk fat from cows housed indoors and fed grass silage- and grass hay-based diets or at pasture (boldface x-axis), over 2 consecutive lactations. Cows received diets containing no additional lipid (control diet, $\mathbf{\square} ; \mathrm{n}=12$ during the first year and $\mathrm{n}=7$ during the second year), or 2.5 to $5.1 \%$ of oil in diet DM from extruded linseeds ( $\cdots \cdots ; n=12$ during the first year and $n=8$ during the second year), extruded rapeseeds $(-\Delta-; \mathrm{n}=12$ during the first year and $\mathrm{n}=4$ during the second year), cold-pressed fat-rich rapeseed meal (--- $\square---; \mathrm{n}=10$ during the first year and $\mathrm{n}=8$ during the second year), or whole unprocessed rapeseeds $(-. . \circ \cdot . ; \mathrm{n}=12$ during the first year and $\mathrm{n}=8$ during the second year). Each point represents treatment LSM and error bars indicate SEM calculated within period (indoor or outdoor; refer to the Materials and Methods section). Effects of treatment, year, and week within year were significant $(P<0.001)$, except for the influence of year on cis-9,trans-11,cis-15 CLnA during outdoor periods $(P=0.48)$, and week within year effect for CLnA cis-9,trans-11,trans-13 CLnA during indoor periods $(P=0.15)$. Treatment by year was only significant $(P<0.001)$ for cis-9,trans-11, cis-15 CLnA during both indoor and outdoor periods. Treatment by week within year was significant $(P<0.05)$ for cis-9,trans-11,cis-15 CLnA during indoor periods, and for cis-9,trans-11,trans-13 CLnA and cis-9,trans-11,trans-15 CLnA during outdoor periods, but not significant $(P>0.20)$ in all other cases. 
Table 2. Effects of oilseed supplements on milk yield, milk fat content and secretion, milk conjugated linoleic acid (CLA) and conjugated linolenic acid (CLnA) composition during consecutive outdoor periods in lactating cows

\begin{tabular}{|c|c|c|c|c|c|c|c|c|c|c|c|c|c|c|}
\hline \multirow[b]{3}{*}{ Item } & \multicolumn{5}{|c|}{ yr $1(2008, \mathrm{n}=58)^{1}$} & \multicolumn{5}{|c|}{ yr $2(2009, \mathrm{n}=35)^{1}$} & \multirow[b]{3}{*}{ SEM } & \multicolumn{3}{|c|}{$P$-value } \\
\hline & \multicolumn{5}{|c|}{ Treatment $^{2}$} & \multicolumn{5}{|c|}{ Treatment $^{2}$} & & \multirow[b]{2}{*}{ Treatment } & \multirow[b]{2}{*}{ Year } & \multirow{2}{*}{$\begin{array}{l}\text { Treatmen } \\
\quad \times \text { year }\end{array}$} \\
\hline & CTL & EL & ER & FRM & WR & CTL & EL & ER & FRM & WR & & & & \\
\hline $\begin{array}{l}\text { Milk yield }(\mathrm{kg} / \mathrm{d}) \\
\text { Milk fat }\end{array}$ & \multicolumn{9}{|c|}{ Milk fat } & & & 0.95 & $<0.001$ & 0.98 \\
\hline Content (\%) & $3.82^{\mathrm{bc}}$ & $4.18^{\mathrm{ab}}$ & $3.83^{\mathrm{bc}}$ & $3.62^{\mathrm{c}}$ & $4.33^{\mathrm{a}}$ & $3.00^{\mathrm{c}}$ & $3.63^{\mathrm{b}}$ & $3.48^{\mathrm{bc}}$ & $3.54^{\mathrm{bc}}$ & $4.24^{\mathrm{a}}$ & 1.85 & 0.01 & $<0.001$ & 0.07 \\
\hline Yield (g/d) & 626 & 708 & 672 & 655 & 746 & 647 & 769 & 717 & 781 & 832 & 57.0 & 0.41 & 0.02 & 0.79 \\
\hline \multicolumn{15}{|c|}{$\begin{array}{l}\text { Concentration }(\mathrm{mg} / 100 \mathrm{~g} \text { of } \mathrm{FA}) \\
\text { of } \mathrm{CLA}^{3} \text { and } \mathrm{CLnA} \mathrm{A}^{4}\end{array}$} \\
\hline cis-9,trans-11 CLA & $2,270^{\mathrm{a}}$ & $1,644^{\mathrm{bc}}$ & $1,456^{\mathrm{bc}}$ & $1,636^{\mathrm{b}}$ & $1,286^{\mathrm{c}}$ & $2,442^{\mathrm{a}}$ & $1,820^{\mathrm{bc}}$ & $1,600^{\mathrm{bc}}$ & $2,148^{\mathrm{b}}$ & $1,395^{\mathrm{c}}$ & 151.9 & $<0.001$ & $<0.001$ & 0.14 \\
\hline cis-11,trans-13 CLA & $2.10^{c}$ & $6.07^{\mathrm{a}}$ & $2.51^{\mathrm{bc}}$ & $3.57^{\mathrm{b}}$ & $2.49^{\mathrm{bc}}$ & $2.59^{\mathrm{c}}$ & $5.57^{\mathrm{a}}$ & $3.03^{\mathrm{bc}}$ & $3.39^{\mathrm{b}}$ & $3.41^{\mathrm{bc}}$ & 0.320 & $<0.001$ & 0.35 & 0.38 \\
\hline cis-12,trans-14 CLA & $0.85^{\mathrm{b}}$ & $4.10^{\mathrm{a}}$ & $1.58^{\mathrm{b}}$ & $1.26^{\mathrm{b}}$ & $0.92^{\mathrm{b}}$ & $1.66^{\mathrm{b}}$ & $5.05^{\mathrm{a}}$ & $2.23^{\mathrm{b}}$ & $1.50^{\mathrm{b}}$ & $2.16^{\mathrm{b}}$ & 0.246 & $<0.001$ & $<0.001$ & 0.62 \\
\hline trans-7,cis-9 CLA & $63.3^{\mathrm{c}}$ & $78.2^{\mathrm{c}}$ & $96.8^{\mathrm{b}}$ & $170.7^{\mathrm{a}}$ & $52.3^{\mathrm{d}}$ & $63.6^{\mathrm{c}}$ & $71.4^{\mathrm{c}}$ & $107.6^{\mathrm{b}}$ & $130.1^{\mathrm{a}}$ & $48.2^{\mathrm{d}}$ & 4.87 & $<0.001$ & $<0.001$ & $<0.001$ \\
\hline trans-9,cis-11 CLA & $17.4^{\mathrm{a}}$ & $14.3^{\mathrm{bc}}$ & $14.9^{\mathrm{bc}}$ & $18.1^{\mathrm{ab}}$ & $12.0^{\mathrm{c}}$ & $20.0^{\mathrm{a}}$ & $15.3^{\mathrm{bc}}$ & $14.2^{\mathrm{bc}}$ & $18.0^{\mathrm{ab}}$ & $10.8^{\mathrm{c}}$ & 1.52 & $<0.01$ & 0.72 & 0.72 \\
\hline trans-10,cis-12 CLA & $6.58^{\mathrm{a}}$ & $3.68^{\mathrm{c}}$ & $4.97^{\mathrm{b}}$ & $8.25^{\mathrm{a}}$ & $4.93^{\mathrm{b}}$ & $8.13^{\mathrm{a}}$ & $3.56^{\mathrm{c}}$ & $6.20^{\mathrm{b}}$ & $6.94^{\mathrm{a}}$ & $6.11^{\mathrm{b}}$ & 0.560 & $<0.001$ & 0.31 & 0.30 \\
\hline trans-11, cis-13 CLA & $44.1^{\mathrm{bc}}$ & $76.3^{\mathrm{a}}$ & $42.7^{\mathrm{c}}$ & $44.5^{\mathrm{bc}}$ & $47.9^{\mathrm{b}}$ & $70.9^{\mathrm{bc}}$ & $96.5^{\mathrm{a}}$ & $57.5^{\mathrm{c}}$ & $76.3^{\mathrm{bc}}$ & $75.3^{\mathrm{b}}$ & 3.55 & $<0.001$ & $<0.001$ & 0.49 \\
\hline trans-12,cis-14 CLA & $4.6^{\mathrm{b}}$ & $34.0^{\mathrm{a}}$ & $7.9^{\mathrm{b}}$ & $7.2^{\mathrm{b}}$ & $6.4^{\mathrm{b}}$ & $7.5^{\mathrm{b}}$ & $40.9^{\mathrm{a}}$ & $11.0^{\mathrm{b}}$ & $8.3^{\mathrm{b}}$ & $8.8^{\mathrm{b}}$ & 1.30 & $<0.001$ & $<0.01$ & 0.28 \\
\hline trans-7,trans-9 CLA & $1.97^{\mathrm{b}}$ & $1.75^{\mathrm{b}}$ & $2.47^{\mathrm{b}}$ & $3.43^{\mathrm{a}}$ & $1.97^{\mathrm{b}}$ & 2.63 & 2.37 & 3.03 & 2.27 & 1.39 & 0.279 & 0.02 & 0.92 & $<0.01$ \\
\hline trans-8,trans-10 CLA & 2.52 & 2.66 & 2.62 & 3.52 & 2.28 & 2.92 & 2.68 & 3.29 & 3.00 & 1.86 & 0.321 & 0.10 & 0.92 & 0.58 \\
\hline trans-9,trans-11 CLA & $19.8^{\mathrm{c}}$ & $34.0^{\mathrm{a}}$ & $18.7^{\mathrm{cd}}$ & $24.5^{\mathrm{b}}$ & $15.8^{\mathrm{d}}$ & $23.1^{\mathrm{b}}$ & $30.8^{\mathrm{a}}$ & $20.7^{\mathrm{b}}$ & $22.8^{\mathrm{b}}$ & $19.8^{\mathrm{b}}$ & 1.30 & $<0.001$ & 0.40 & 0.06 \\
\hline trans-10,trans-12 CLA & $7.32^{\mathrm{b}}$ & $6.82^{\mathrm{c}}$ & $7.15^{\mathrm{bc}}$ & $12.86^{\mathrm{a}}$ & $4.27^{\mathrm{d}}$ & $8.89^{\mathrm{b}}$ & $6.33^{\mathrm{c}}$ & $8.68^{\mathrm{bc}}$ & $11.30^{\mathrm{a}}$ & $5.34^{\mathrm{d}}$ & 0.549 & $<0.001$ & 0.35 & 0.12 \\
\hline trans-11,trans-13 CLA & $42.8^{\mathrm{c}}$ & $140.6^{\mathrm{a}}$ & $59.1^{\mathrm{c}}$ & $98.8^{\mathrm{b}}$ & $50.2^{\mathrm{c}}$ & $56.4^{\mathrm{c}}$ & $134.4^{\mathrm{a}}$ & $65.6^{\mathrm{bc}}$ & $82.6^{\mathrm{b}}$ & $65.6^{\mathrm{bc}}$ & 5.44 & $<0.001$ & 0.50 & 0.04 \\
\hline trans-12,trans-14 CLA & $17.2^{\mathrm{d}}$ & $69.1^{\mathrm{a}}$ & $23.7^{\mathrm{c}}$ & $35.0^{\mathrm{b}}$ & $21.4^{\mathrm{cd}}$ & $19.7^{\mathrm{c}}$ & $62.8^{\mathrm{a}}$ & $24.5^{\mathrm{bc}}$ & $28.9^{\mathrm{b}}$ & $24.9^{\mathrm{bc}}$ & 2.04 & $<0.001$ & 0.45 & 0.07 \\
\hline trans-13,trans-15 CLA & $0.65^{\mathrm{c}}$ & $2.70^{\mathrm{a}}$ & $1.10^{\mathrm{bc}}$ & $1.21^{\mathrm{b}}$ & $1.00^{\mathrm{bc}}$ & $1.18^{\mathrm{c}}$ & $3.31^{\mathrm{a}}$ & $1.28^{\mathrm{bc}}$ & $1.40^{\mathrm{b}}$ & $1.45^{\mathrm{bc}}$ & 0.144 & $<0.001$ & $<0.001$ & 0.40 \\
\hline$\Sigma \mathrm{CLA}^{3}$ & $2,529^{\mathrm{a}}$ & $2,131^{\mathrm{bc}}$ & $1,762^{\mathrm{cd}}$ & $2,089^{\mathrm{ab}}$ & $1,525^{\mathrm{d}}$ & $2,754^{\mathrm{a}}$ & $2,309^{\mathrm{bc}}$ & $1,938^{\text {cd }}$ & $2,569^{\mathrm{ab}}$ & $1,683^{\mathrm{d}}$ & 158.2 & $<0.001$ & $<0.001$ & 0.33 \\
\hline $\begin{array}{l}\text { cis-9,trans-11,cis- } 15 \\
\text { CLnA }\end{array}$ & $70.0^{\mathrm{b}}$ & $225.6^{\mathrm{a}}$ & $85.5^{\mathrm{b}}$ & $66.8^{\mathrm{b}}$ & $83.3^{\mathrm{b}}$ & $86.6^{\mathrm{b}}$ & $215.8^{\mathrm{a}}$ & $93.6^{\mathrm{b}}$ & $79.7^{\mathrm{b}}$ & $107.0^{\mathrm{b}}$ & 10.34 & $<0.001$ & 0.06 & 0.28 \\
\hline $\begin{array}{l}\text { cis-9,trans-11,trans-13 } \\
\text { CLnA }\end{array}$ & $25.7^{\mathrm{c}}$ & $53.2^{\mathrm{a}}$ & $32.1^{\mathrm{bc}}$ & $38.2^{\mathrm{b}}$ & $27.0^{c}$ & $28.6^{\mathrm{c}}$ & $60.0^{\mathrm{a}}$ & $33.9^{\mathrm{bc}}$ & $37.2^{\mathrm{b}}$ & $27.4^{\mathrm{c}}$ & 2.76 & $<0.001$ & 0.28 & 0.72 \\
\hline $\begin{array}{l}\text { cis-9,trans-11,trans- } 15 \\
\text { CLnA }\end{array}$ & $55.7^{\mathrm{ab}}$ & $61.3^{\mathrm{a}}$ & $44.8^{\mathrm{b}}$ & $43.7^{\mathrm{b}}$ & $46.6^{\mathrm{b}}$ & $48.8^{\mathrm{ab}}$ & $61.5^{\mathrm{a}}$ & $37.5^{\mathrm{b}}$ & $52.9^{\mathrm{b}}$ & $48.8^{\mathrm{b}}$ & 4.20 & 0.02 & 0.88 & 0.48 \\
\hline$\Sigma \mathrm{CLnA}^{4}$ & $143.5^{\mathrm{b}}$ & $340.4^{\mathrm{a}}$ & $162.7^{\mathrm{b}}$ & $148.7^{\mathrm{b}}$ & $157.1^{\mathrm{b}}$ & $165.3^{\mathrm{b}}$ & $343.2^{\mathrm{a}}$ & $168.5^{\mathrm{b}}$ & $169.7^{\mathrm{b}}$ & $184.3^{\mathrm{b}}$ & 12.70 & $<0.001$ & 0.08 & 0.85 \\
\hline
\end{tabular}

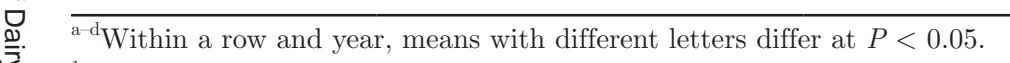

@ ${ }^{1}$ Measured on 1 occasion per year of experiment during wk $41 \pm 3.3$ and $37 \pm 5.0$ (means \pm SD) of lactation for yr 1 and 2 , respectively.

$\stackrel{\circ}{\circ}$. $\quad{ }^{2}$ Cows fed a pasture-based diet containing no additional oilseed supplement (CTL; $\mathrm{n}=12$ and 7 during yr 1 and 2 , respectively), or $2.5 \%$ of oil in diet DM from extruded linseeds $(\mathrm{EL} ; \mathrm{n}=12$ and 8 during yr 1 and 2 , respectively), extruded rapeseeds (ER; $\mathrm{n}=12$ and 4 during yr 1 and 2 , respectively), cold-pressed fat-rich rapeseed meal (FRM; $\mathrm{n}=10$ and 8 during yr 1 and 2, respectively), or whole unprocessed rapeseeds (WR; $\mathrm{n}=12$ and 8 during yr 1 and 2, respectively).

으 ${ }^{3}$ Determined by GC and HPLC.

co $\quad{ }^{4}$ Determined by GC. 
and CLnA concentrations were higher $(P<0.001)$ for ER and EL compared with the CTL (Table 1). Relative to ER, EL resulted in increased $(P<0.001)$ enrichment of cis-9,trans-11 CLA, trans-11,cis-13 CLA, trans-11,trans-13 CLA, trans-12,trans-14 CLA, trans-13,trans-15 CLA, and cis-9,trans-11,cis-15 CLnA (Table 1; Figures 2C and 3A). Conversely, trans-7, cis-9 CLA was increased to a greater $(P<0.001)$ extent by ER compared with EL. Furthermore, EL during the first indoor period increased $(P<0.001)$ cis-11,trans-13 CLA, cis-12,trans-14 CLA, trans-12,cis-14 CLA, trans9,trans-11 CLA, and cis-9,trans-11,trans-15 CLnA (Table 1 and Figure 3C).

At pasture, both EL and ER decreased $(P<0.001)$ total milk fat CLA concentration compared with the CTL, whereas EL increased $(P<0.001)$ total CLnA concentration (Table 2). Dietary EL supplement specifically increased $(P<0.001) \Delta 11,13$ CLA, cis12,trans-14 CLA, trans-12,cis-14 CLA, trans-9,trans-11 CLA, trans-13,trans-15 CLA, $\Delta 9,11,15$ CLnA, and cis-9,trans-11,trans-13 CLnA (Table 2 and Figure 3). Relative to the CTL, EL and ER to a lesser extent, increased $(P<0.001)$ milk fat trans-12,trans-14 CLA concentration (Table 2). Enrichment of CLA isomers by EL were, in most cases, of higher magnitude at pasture than during the indoor period $(P<0.001$; data not shown), whereas the reverse was true for trans-11,cis-13 CLA, trans-13,trans-15 CLA, and $\Delta 9,11,15$ CLnA. At pasture, ER, but not EL, increased $(P<0.001)$ milk trans-7,cis-9 CLA concentration (Table 2), but the magnitude of response was lower than during the indoor period $(P<0.001$; data not shown). In contrast to the indoor period, both EL and ER lowered $(P<$ 0.001) milk fat cis-9,trans-11 CLA, trans-9,cis-11 CLA, and trans-10,cis-12 CLA concentrations relative to the CTL (Table 2; Figures 2C, 2D, and 2F).

Effects of Rapeseed Form (ER vs. FRM vs. WR) During yr 1. During the first indoor period, ER and FRM increased $(P<0.001)$ to a similar extent total milk fat CLA concentration compared with the CTL, whereas WR had no effect (Table 1). All rapeseed treatments increased $(P<0.001)$ total CLnA concentration, with responses to ER being higher $(P<0.001)$ than to FRM and WR. Both ER and FRM resulted in similar increases $(P<0.001)$ in milk cis-9,trans-11 CLA, trans-11,trans-13 CLA, and trans-13,trans-15 CLA concentrations (Table 1; Figure 2C). However, increases in trans-7,cis-9 CLA and trans-12,trans-14 CLA concentrations were greater $(P<0.001)$ for FRM than for ER. Changes in milk conjugated FA to WR were confined to increases $(P<0.001)$ in milk trans11,cis-13 CLA and cis-9,trans-11,cis-15 CLnA concentration, responses that were similar in magnitude to the increases induced by ER (Table 1 and Figure 3A).
At pasture, ER and WR in particular, decreased $(P$ $<0.001)$ milk total CLA concentration but had no effect $(P>0.10)$ on total CLnA concentration (Table 2). Consistent with the effects observed during the indoor period, FRM increased milk trans-7, cis-9 CLA concentration to a greater extent than ER, whereas only FRM induced increases $(P<0.001)$ in trans-13,trans-15 CLA (Table 2). However, milk trans-12,trans-14 CLA concentration was increased more by FRM than ER, whereas FRM was the only rapeseed treatment to enrich trans11,trans-13 CLA, cis-11,trans-13 CLA, trans-7,trans-9 CLA, trans-9,trans-11 CLA, trans-10,trans-12 CLA, and cis-9,trans-11,trans-13 CLnA (Table 2 and Figure $3 \mathrm{~B} ; P<0.001)$. Increases in these specific CLA isomers by FRM were of higher magnitude at pasture when compared with the indoor period $(P<0.001$; data not shown), with the exception of trans-13,trans-15 CLA, for which the increase was lower $(P<0.001)$. In contrast to the effects during the indoor period, all of the rapeseed treatments, WR in particular, lowered $(P<$ 0.001; data not shown) milk fat cis-9,trans-11 CLA concentration at pasture (Table 2; Figure 2C). Furthermore, WR also decreased $(P<0.001)$ trans-7, cis-9 CLA, trans-9,trans-11 CLA, and trans-10,trans- 12 CLA, whereas both ER and WR decreased $(P<0.001)$ milk trans-9,cis-11 CLA and trans-10,cis-12 CLA content during the outdoor period (Table 2; Figures 2D and $2 \mathrm{~F})$.

Interactions Between Treatment and Year. Interactions between treatment and year were significant $(P<0.05)$ for the concentration of 12 out of 18 conjugated FA in milk from the indoor periods (Table 1). Compared with the CTL, increases in milk total CLA and cis-9,trans-11 CLA concentrations were lower for EL, but higher to FRM during the second compared with the first indoor period. Changes in total CLA and cis-9,trans-11 CLA by ER were not affected by year of study, whereas responses to WR were lower in the second than first indoor feeding period (Table 1; Figure $2 \mathrm{C} ; P<0.001)$. Treatments EL and ER resulted in a higher enrichment of trans-7, cis-9 CLA; EL increased cis-11,trans-13 CLA, trans-12,cis-14 CLA, and trans13,trans-15 CLA; and FRM enhanced trans-9,trans-11 CLA, and trans-11,trans-13 CLA to a greater extent during the second than the first indoor period (Table $1 ; P<0.001)$. In contrast, EL induced lower increases in milk cis-12,trans-14 CLA, trans-11,cis-13 CLA, and cis-9,trans-11,cis-15 CLnA concentrations during the second than the first indoor period (Table 1 and Figure 3A; $P<0.001$ ). During the second indoor period, milk trans-9,cis-11 CLA and trans-10,cis-12 CLA concentrations were increased by EL, but decreased $(P<$ 0.001) in response to WR (Table 1 and Figures $2 \mathrm{D}$ and $2 \mathrm{~F})$. Furthermore, EL, ER, and FRM lowered trans- 
8,trans-10 CLA abundance, and both ER and WR decreased milk trans-10,trans-12 CLA concentration (Table $1 ; P<0.001$ ). Irrespective of study year, oilseed treatments induced similar changes in cis-9, trans11,trans-13 CLnA and cis-9,trans-11,trans-15 CLnA concentrations in cows fed conserved forages (Table 1 and Figures 3B and 3C).

The effects of oilseed treatments were generally similar between both outdoor periods (Table 2; interaction treatment by year: $P>0.10$ for 13 out of 18 conjugated FA). However, milk trans-7,cis-9 CLA was increased to a greater or lesser extent by ER or FRM, respectively, whereas increases in milk trans-11,trans-13 CLA and trans-12,trans-14 CLA by EL and FRM were marginally lower during the second than the first outdoor period (Table $2 ; P<0.10$ ). In contrast to the first outdoor period, the abundance of trans-9,trans-11 CLA was not affected by FRM and WR and only slightly increased by EL at pasture in yr 2 .

\section{Relationships Between Milk CLA, CLnA, and Other FA}

Pairwise Correlation and Regression Analyses. Irrespective of period (indoor or outdoor), a close association existed between trans-11,cis-13 CLA and trans-11,trans-15 18:2 ( $\mathrm{r}=0.88 ; P<0.001 ; \mathrm{n}=186)$, and between trans-12,cis-14 CLA and cis-15 18:1 concentrations $(\mathrm{r}=0.79 ; P<0.001 ; \mathrm{n}=186)$. Significant $(P<0.001)$ pairwise correlation coefficients above 0.70 also existed between milk trans-12,cis-14 CLA, trans-11,trans-13 CLA, trans-12,trans-14 CLA, and trans-13,trans-15 CLA concentrations for both periods ( $\mathrm{n}=93$ for each period). Milk fat trans-11,trans-13 CLA, trans-12,trans-14 CLA, and trans-13,trans-15 CLA concentrations were closely correlated with cis-15 18:1, trans-12 18:1, trans-13,-14 18:1, trans-16 18:1, cis9,trans-13 18:2, and cis-9,trans-14 18:2 concentrations ( $\mathrm{r}>0.70 ; P<0.001 ; \mathrm{n}=186)$, but within each period, only the associations between trans-11,trans-13 CLA and trans-12,trans-14 CLA with trans-12 18:1 remained significant $(\mathrm{r}>0.67 ; P<0.001 ; \mathrm{n}=93$ for each period). Variations in milk trans-9,trans-11 CLA concentrations were closely correlated with changes in cis-12 18:1, cis9,trans-13 18:2, and cis-9,trans-14 18:2 ( $\mathrm{r}>0.69 ; P<$ $0.001 ; \mathrm{n}=186$ ). Milk fat trans-10,trans-12 CLA concentration was closely associated with trans-10 18:1 for all periods ( $\mathrm{r}>0.70 ; P<0.001 ; \mathrm{n}=93$ for each period). Strong associations existed between trans-10, cis-12 CLA and trans-9,cis-11 CLA with trans-10 18:1 concentration in milk produced during indoor feeding periods $(P<0.001 ; \mathrm{r}>0.65$ and $\mathrm{r}<0.40$ during the indoor and outdoor periods, respectively; $\mathrm{n}=93$ ).
Close linear relationships were identified between milk cis-9,trans-11 CLA and trans-11 18:1 concentration, and between trans-7,cis-9 CLA and trans-6,-7,-8 18:1 concentrations (Figures 4A and B; $\mathrm{r}>0.91 ; P<$ $0.001 ; \mathrm{n}=186)$. Significant associations also existed between milk cis-9,trans-11,trans-13 CLnA and trans11,trans-13 CLA concentrations, and between cis9,trans-11,trans-15 CLnA and trans-11,trans-15 18:2 concentrations (Figures $4 \mathrm{C}$ and $4 \mathrm{D} ; \mathrm{r}=0.77$ and 0.70 , respectively; $P<0.001 ; \mathrm{n}=186$ and 465 , respectively). However, much weaker correlation coefficients existed between cis-9,trans-11, cis-15 CLnA and trans-11,cis-15 18:2 concentrations (Figure 4E; $\mathrm{r}=0.56 ; P<0.001 ; \mathrm{n}$ $=465)$, which were often not significant within treatment $(\mathrm{r}=+0.28,-0.09,+0.27,+0.15$, and $+0.28 ; P=$ $0.01,0.37,0.01,0.15$, and $0.01 ; \mathrm{n}=95,100,80,90$, and 100 for CTL, EL, ER, FRM, and WR, respectively).

PCA on Milk from the EL Treatment. The PCA enabled major associated changes in the concentration of 18-carbon FA in milk samples $(\mathrm{n}=40)$ from cows fed the EL treatment to be investigated and summarized. The first and second principal components (PC1 and PC2, respectively, Figure 5A) accounted for 55\% of the total variance. The PC1 discriminates cis-11 18:1, trans11,trans-15 18:2, cis-12,trans-14 CLA, trans-11,cis-13 CLA, and cis-9,trans-11,cis-15 CLnA (positively), from cis-12 18:1, cis-13 18:1, and cis-15 18:1, all trans 18:1 isomers (other than trans-11 18:1), nonconjugated 18:2 (except trans-11,trans-15 18:2), trans-7,cis-9 CLA, trans-9,cis-11 CLA, trans-10,cis-12 CLA, and 18:3n-3 (negatively). In addition, PC1 was positively or negatively associated with milk fat content or dietary starch intake, respectively. The $\mathrm{PC} 2$ had a positive loading for the sum of even-chain FA (4:0 to 14:0), and a negative loading for cis-11 18:1, trans-6,-7,-8 18:1, trans-11 18:1, cis-9,trans-11 CLA, cis-11,trans-13 CLA, trans12,cis-14 CLA, trans-9,trans-11 CLA, trans-10,trans-12 CLA, trans-11,trans-13 CLA, trans-12,trans-14 CLA, and cis-9,trans-11,trans-13 CLnA.

Figure 5B illustrates the location of all 40 samples of milk on the EL treatment during the indoor and outdoor periods for yr 1 and 2 on PC1 and PC2. The $\mathrm{PC} 1$ discriminates milk from the first indoor period and both outdoor periods (enriched in trans-11,trans-15 18:2, cis-12,trans-14 CLA, trans-11,cis-13 CLA, and cis-9,trans-11,cis-15 CLnA), from those of the second indoor period [enriched in trans 18:1 and trans 18:2 (except trans-11 18:1 and trans-11,trans-15 18:2), 18:2n-6, trans-7,cis-9 CLA, trans-9,cis-11 CLA, trans-10,cis-12 CLA, and 18:3n-3]. Along the PC2, samples of milk for $\mathrm{EL}$ in the first indoor period are discriminated from the milk samples collecting during outdoor periods (enriched in $\Delta 11$ 18:1, cis-9,trans-11 CLA, cis-11,trans-13 CLA, trans-12,cis-14 CLA, and trans,trans CLA with 

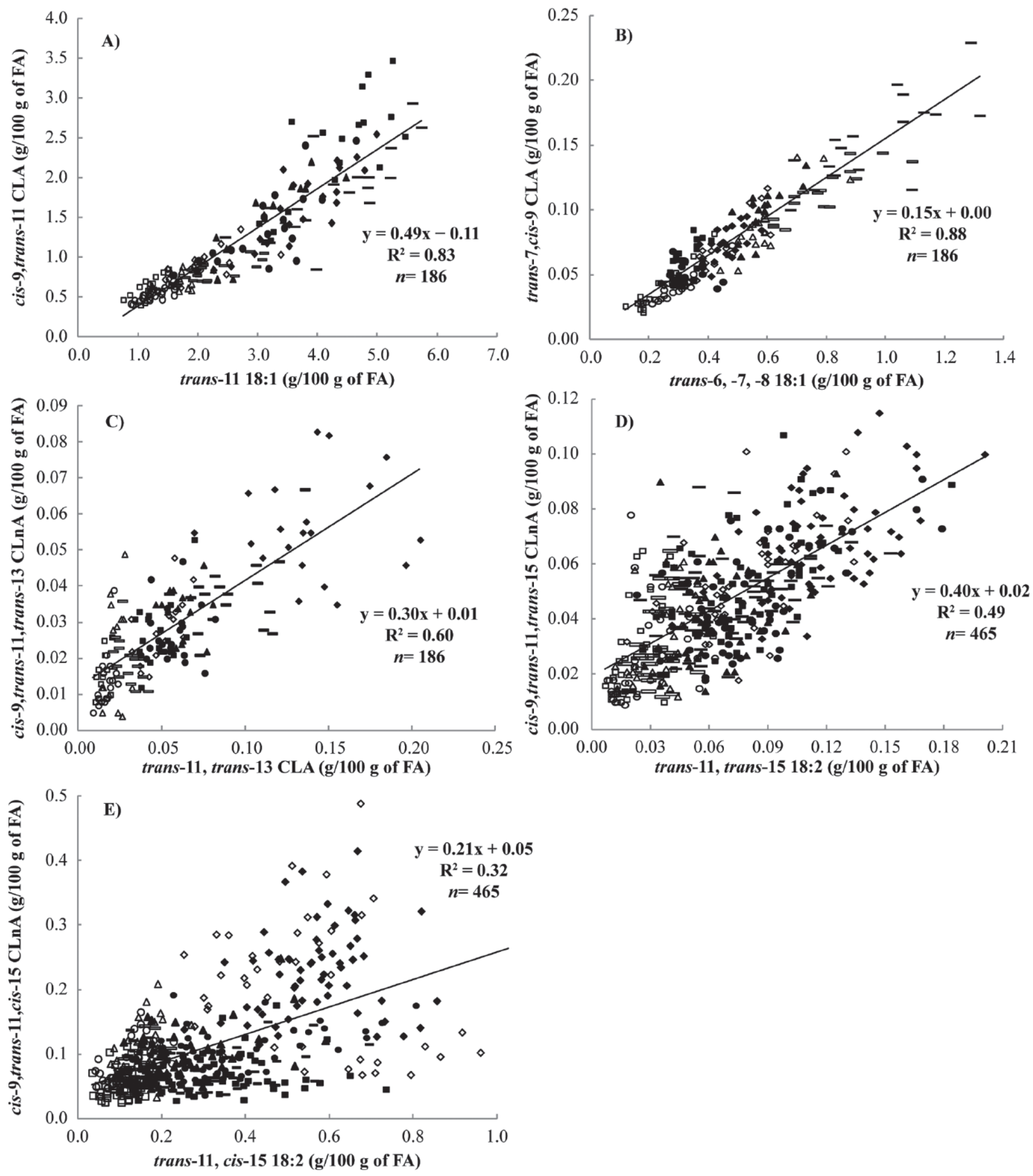

Figure 4. Relationships between (A) trans-11 18:1 and cis-9,trans-11 conjugated linoleic acid (CLA), (B) trans-6, -7, -8 18:1 and trans7,cis-9 CLA, (C) trans-11,trans-13 CLA and cis-9,trans-11,trans-13 conjugated linolenic acid (CLnA), (D) trans-11,trans-15 18:2 and cis-9,trans11,trans-15 CLnA, and (E) trans-11,cis-15 18:2 and cis-9,trans-11,cis-15 CLnA concentrations in milk fat from cows housed indoors and fed grass silage- and grass hay-based diets (open symbols) or at pasture (filled symbols), over 2 consecutive lactations. $P<0.001$ in all cases. Cows received diets containing no additional lipid (control diet, $\square$ and $\mathbf{\square} ; \mathrm{n}=12$ during the first year and $\mathrm{n}=7$ during the second year), or 2.5 to $5.1 \%$ of oil in diet DM from extruded linseeds ( $\diamond$ and $\diamond ; \mathrm{n}=12$ during the first year and $\mathrm{n}=8$ during the second year), extruded rapeseeds $(\Delta$ and $\boldsymbol{\Delta} ; \mathrm{n}=12$ during the first year and $\mathrm{n}=4$ during the second year), cold-pressed fat-rich rapeseed meal $(=$ and $\boldsymbol{-} ; \mathrm{n}=10$ during the first year and $n=8$ during the second year), or whole unprocessed rapeseeds ( $O$ and $\mathbf{0} ; n=12$ during the first year and $n=8$ during the second year). 

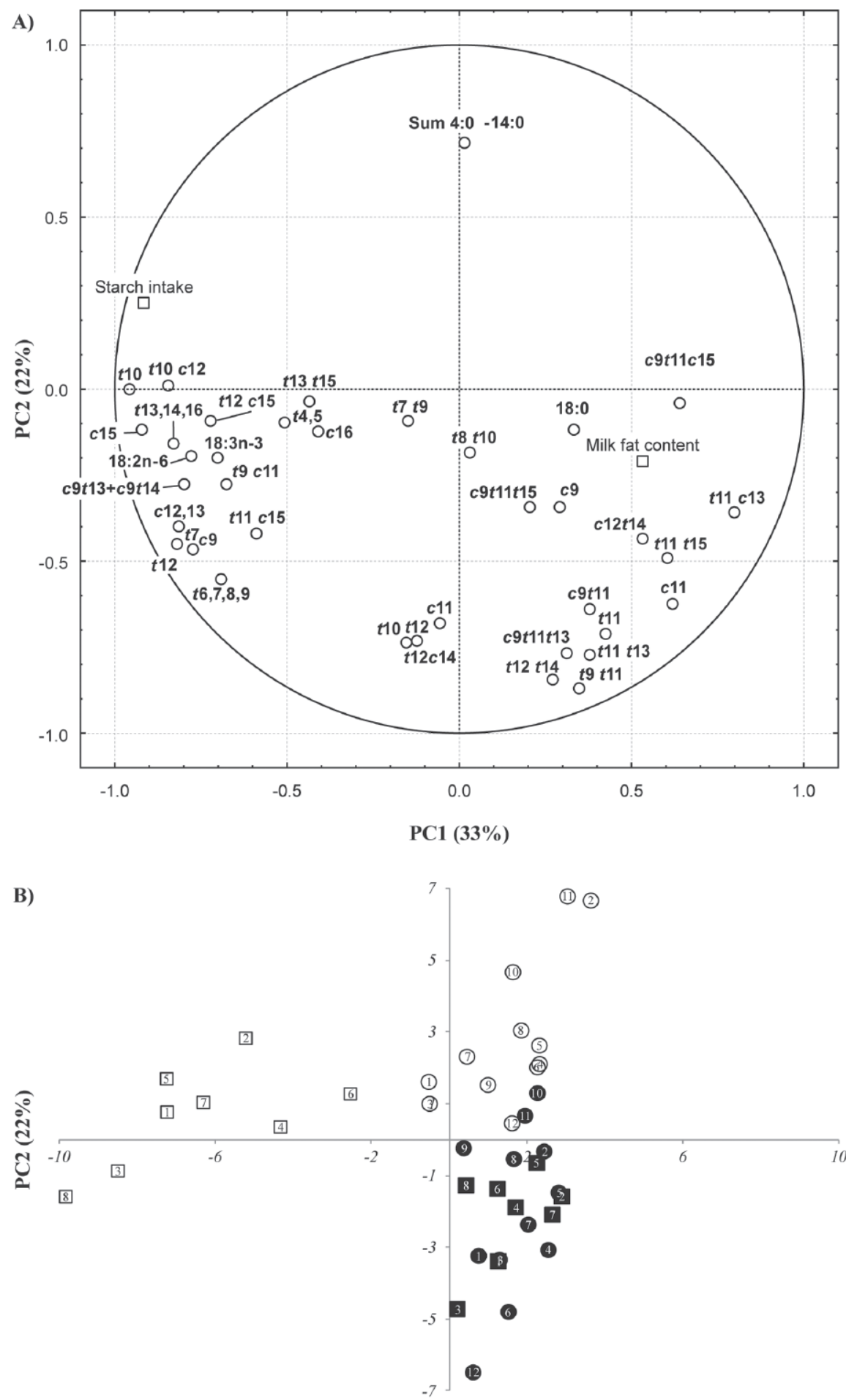

PC1 (33\%)

Figure 5. Loading plot of principal components (PC) 1 and 2 that accounted for 33 and $22 \%$, respectively, of total variance in the concentrations of $36 \mathrm{FA}$ with 18-carbons determined in 40 milk samples from cows housed indoors and fed grass silage- and grass hay-based diets or at pasture, and receiving 2.5 to $3.5 \%$ oil in diet DM from extruded linseeds over 2 consecutive lactations (12 cows during the first year and 8 cows during the second year). (A) Plots of variables are projected on the first 2 PC. $c \Delta$ and $t \Delta$ represent cis and trans positional isomers, respectively, and non-boldface data indicate variables that did not participate in the PC (i.e., milk fat content and starch intake). (B) Plots of milks (cows individually identified as 1 to 12) on the first 2 PC: indoor periods (open symbols) during the first (O) or second year ( $\square$ ), or outdoor periods (filled symbols) during the first $(-)$ and second year $(\boldsymbol{\square})$. Effects due to period (indoor vs. outdoor) and year for indoor periods were significant for the $2 \mathrm{PC}(P<0.01)$, but the effect due to year during outdoor periods was not significant $(P>0.20)$. 
double bonds from $\Delta 9,11$ to $\Delta 12,14$, and cis-9,trans11,trans-13 CLnA), whereas milks from the indoor period in yr 2 are intermediate. None of the $2 \mathrm{PC}$ allowed for the discrimination of samples of EL milk produced during the first versus the second outdoor period.

\section{Relationships Between Milk Fat Content or Yield, with trans FA Concentrations}

Relationships between milk fat content or yield, and concentrations of intermediates of ruminal biohydrogenation (RBH) were studied based on 20 comparisons derived from differences between each treatment least squares means $(\mathrm{n}=5)$ and the mean of the 5 treatment least squares means within each of the 4 feeding periods (Figure 6). The highest differences between 2 treatment least squares means were observed during the second outdoor period for milk fat content (difference of $1.24 \mathrm{~g}$ of fat/100 g of milk between CTL and WR) and during the second indoor period for milk fat yield (difference of $273 \mathrm{~g} / \mathrm{d}$ between ER and WR). Among all identified 18-carbon FA, variations in milk trans-10 18:1, trans9,cis-11 CLA, and trans-10,cis-12 CLA concentrations exhibited the most significant associations with changes in mean milk fat concentration (Figure $6, \mathrm{r}=-0.83$, -0.69 , and $-0.64 ; P<0.001)$ and milk fat yield $(\mathrm{r}=$ $-0.72,-0.69$, and $-0.42 ; P<0.001, P<0.001$, and $P=0.06$ for trans-9,cis-11 CLA, trans-10,cis-12 CLA, and trans-10 18:1, respectively).

\section{DISCUSSION}

Relatively few experiments have examined the effect of oilseed supplements on the abundance of specific isomers of CLA and CLnA in bovine milk, but none have examined the persistency of the response over an extended period. Novel aspects of the present investigation include a detailed assessment of the effect of dietary oilseed supplements on milk CLA and CLnA composition in cows fed conserved forages in the winter and pasture in the summer over 2 consecutive lactations.

\section{Origin of CLA and CLnA in Milk Fat}

Previous investigations have reported the occurrence of cis-9,trans-11,cis-15 CLnA and cis-9,trans-11,trans-15 CLnA in ruminant milk (Plourde et al., 2007; GómezCortés et al., 2009; Halmemies-Beauchet-Filleau et al., 2011), but this is the first report to confirm the appearance of the fully conjugated $\alpha$-eleostearic acid (cis-9,trans-11,trans-13 CLnA) in bovine milk. Studies in animal models have provided some evidence that $\alpha$-eleostearic acid exhibits antiinflammatory, immuno- modulatory, antiobesity, and anticarcinogenic activities when fed at low (0.05 to $1 \%$ of total dietary intake) concentrations (Koba et al., 2007, Hennessy et al., 2011). However, the concentration of cis-9,trans-11,trans-13 CLnA in bovine milk (mean $0.025 \mathrm{~g} / 100 \mathrm{~g}$ of FA, 8.3 $\mathrm{mg} / \mathrm{kg}$ of milk, $\mathrm{n}=465$; maximum of $0.083 \mathrm{~g} / 100 \mathrm{~g}$ of FA, $28.0 \mathrm{mg} / \mathrm{kg}$ of milk) appears probably too low to be of significant benefit in humans.

Several investigations have shown that the majority of cis-9,trans-11 CLA secreted in bovine milk originates from the action of the $\Delta^{9}$-desaturase on trans-11 18:1 in the mammary gland (Griinari et al., 2000; Palmquist et al., 2005). A close relationship between milk fat trans-11 18:1 and cis-9,trans-11 CLA in milk of cows fed a range of oilseed supplements in this study (Figure $4 \mathrm{~A}$ ) is consistent with most of the cis-9,trans-11 CLA in milk being synthesized endogenously. Similarly, milk fat trans-7,cis-9 CLA and trans-6,-7,-8 18:1 concentrations were closely correlated (Figure 4B) with a small positive intercept. This relationship suggests that trans-7,cis-9 CLA in milk originates almost exclusively from the action of $\Delta^{9}$-desaturase on trans-7 18:1 in the mammary gland. These findings are consistent with postruminal infusions of sterculic oil or trans-10, cis-12 CLA, known to inhibit mammary $\Delta^{9}$-desaturase activity, lowering milk fat trans-7,cis-9 CLA concentration (Corl et al., 2002) and earlier reports that omasal or duodenal digesta is devoid of trans-7,cis-9 CLA (Palmquist et al., 2005). The slope of the relationship between milk trans-7, cis-9 CLA and trans-6,-7,-8 18:1 concentrations was lower than that between milk cis-9,trans-11 CLA and trans-11 18:1 concentrations. Differences in the slope of these relationships do not directly infer a lower specificity of $\Delta^{9}$-desaturase for trans-7 18:1 than trans-11 18:1, but are most likely explained by the inability to resolve trans-7 18:1 as a single peak during GC analysis. Recording of the mass spectrum of the DMOX derivative in selected samples corresponding to unresolved trans-6,-7,-8 18:1 during GC analysis of FAME confirmed that trans-6 18:1 was a major component of the coeluting trans-6,-7,-8 18:1 peak.

Milk cis-9,trans-11,trans-13 CLnA and cis-9,trans11,trans-15 CLnA concentrations were closely correlated with trans-11,trans-13 CLA and trans-11,trans-15 18:2 concentrations, respectively (Figures 4C and 4D). These findings also suggest that both cis-9,trans11,trans-13 CLnA and cis-9,trans-11,trans-15 CLnA in milk are synthesized endogenously via the action of $\Delta^{9}$-desaturase on trans-11,trans-13 CLA and trans11,trans-15 18:2 in the mammary gland, respectively. Such a hypothesis is supported by the apparent absence of cis-9,trans-11,trans-13 CLnA and cis-9,trans11,trans-15 CLnA during incubations of 18:3n-3 with 
rumen fluid or in digesta leaving the rumen (Palmquist et al., 2005; Wąsowska et al., 2006; Shingfield et al., 2010). A relatively weak association between milk cis9,trans-11,cis-15 CLnA and trans-11,cis-15 18:2 concentrations, as well as the low slope of the regression between these variables (Figure $4 \mathrm{E}$ ) are consistent with cis-9,trans-11,cis-15 CLnA secreted in milk originating predominantly from the rumen rather than endogenous synthesis in the mammary gland. Indeed, several studies have shown that cis-9,trans-11,cis-15 CLnA is formed during the biohydrogenation of $18: 3 n-3$ in vitro and is present in omasal digesta (Palmquist et al., 2005; Wąsowska et al., 2006; Shingfield et al., 2010). Although cis-11 18:1 was relatively abundant in milk fat (mean $0.82 \mathrm{~g} / 100 \mathrm{~g}$ of FA; Lerch et al., 2012b), only $53 \%$ of the 186 analyzed samples contained cis-9,cis-11 CLA in even trace amounts (average of $2.1 \mathrm{mg} / 100 \mathrm{~g}$ of FA). Such findings could be interpreted as evidence that cis 18:1 and 18:2 isomers are less-preferred substrates for mammary $\Delta^{9}$-desaturase compared with trans unsaturated FA, supporting much earlier observations based on FA incubations with rat liver microsomal preparations (Pollard et al., 1980).

\section{Effects of Indoor Versus Outdoor Period}

With few exceptions (trans-10,cis-12 CLA, trans7,trans-9 CLA, and trans-8,trans-10 CLA), milk produced on the CTL contained higher amounts of total and all measured isomers of CLA and CLnA at pasture compared with indoor feeding periods (on average, for the $2 \mathrm{yr}$, relative increases of 240 and $82 \%$ in total CLA and total CLnA, respectively). It is well established that milk at pasture contains higher concentrations of total CLA compared with milk produced from diets containing high proportions of grass silage or dried grass (Mohammed et al., 2009), corn silage (Couvreur et al., 2006), or concentrates (Kraft et al., 2003).

In the present study, the increase in milk CLA and CLnA concentrations at pasture are not solely explained by marginally higher dietary $18: 2 \mathrm{n}-6$ and $18: 3 \mathrm{n}-$ 3 concentration during outdoor than indoor periods (on average, for the $2 \mathrm{yr}$, relative differences in the sum of $18: 2 \mathrm{n}-6$ and $18: 3 \mathrm{n}-3$ in the diet estimated to be $14 \%$ higher). The underlying reasons for the higher enrichment of conjugated FA in milk from pasture compared with conserved forages are not fully understood, but may be related to the relatively high amount of rapidly fermentable sugars, soluble fiber, or possibly certain metabolites such as polyphenols and terpenes in fresh grass, higher rumen passage rate and fluid dilution rate in cows fed fresh grass, or possibly due to feeding behavior (meal size, feeding frequency, and rumination; Kraft et al., 2003; Mohammed et al., 2009).
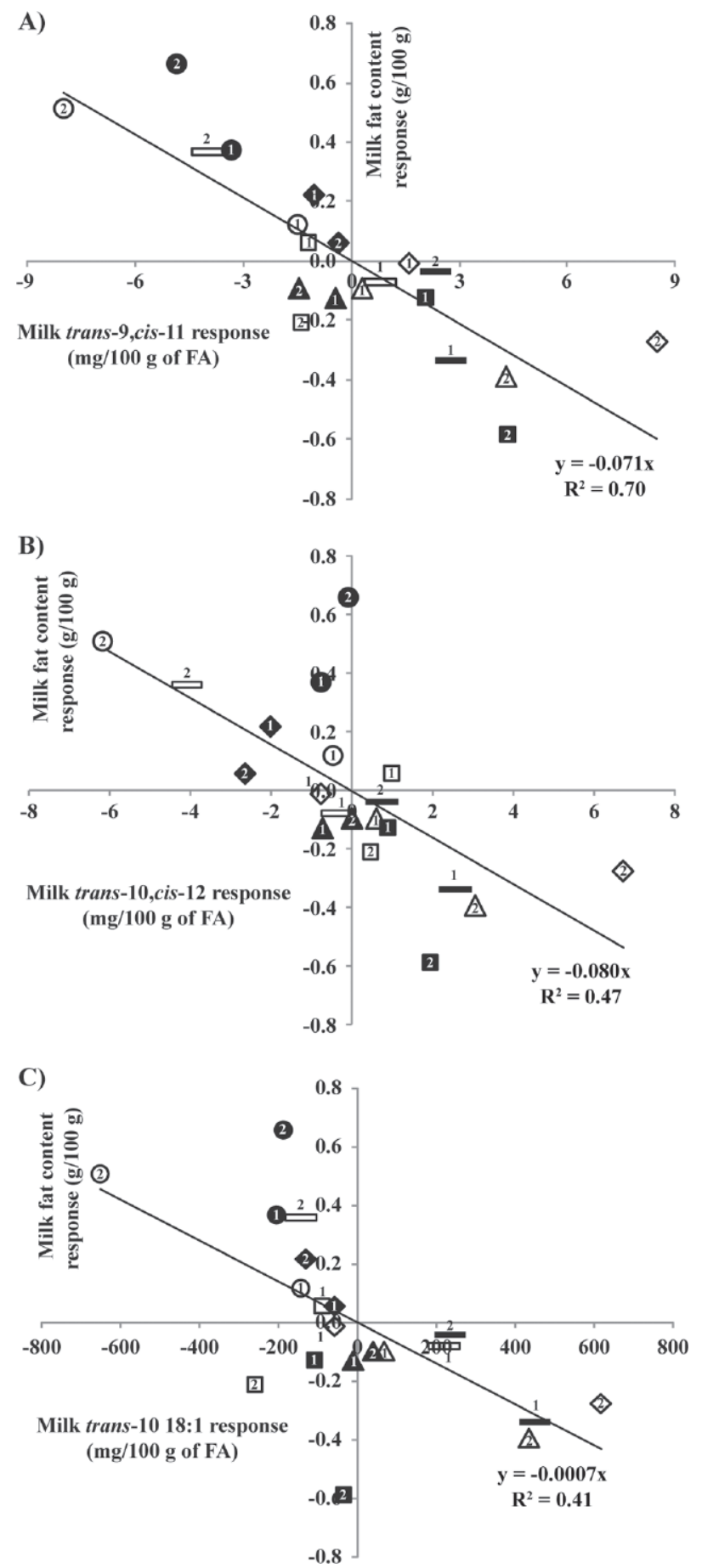

Figure 6. Relationships between milk responses of (A) trans9,cis-11 conjugated linoleic acid (CLA) and fat content, (B) trans10,cis-12 CLA and fat content, (C) trans-10 18:1 and fat content (n $=20$ ). Each value represents the difference between LSM for each treatment and average of the 5 treatment LSM within each period. Cows were housed indoors and fed grass silage- and grass hay-based diets (open symbols) or at pasture (filled symbols), over 2 consecutive lactations (year indicated as 1 or 2 ). Cows received diets containing no additional lipid ( $\square$ and $\mathbf{\square}$, control diet), or 2.5 to $5.1 \%$ of oil in diet DM from extruded linseeds ( $\diamond$ and $\boldsymbol{\diamond})$, extruded rapeseeds ( $\Delta$ and $\boldsymbol{\Delta})$, cold-pressed fat-rich rapeseed meal $(=$ and $\boldsymbol{- a})$, or whole unprocessed rapeseeds ( $\bigcirc$ and $\bullet$ ). 


\section{Effects of Oilseed Supplements During Indoor Periods}

Effects of Oilseed Nature (EL vs. ER) During $y r$ 1. Supplements of EL and ER altered the concentration and isomer distribution of CLA in milk fat, which differed between treatments. Treatment EL resulted in a higher enrichment of $\Delta 11,13$ CLA and $\Delta 12,14$ CLA in milk, whereas ER caused a greater increase in milk trans-7,cis-9 CLA concentration. Similar differences in milk fat CLA responses due to oilseed nature have been reported in cows fed diets based on hay or grass silage supplemented with 2.3 to $2.6 \%$ of added oil in DMI from ground rapeseeds and linseeds (Collomb et al., 2004) or rapeseed and linseed oil (Shingfield et al., 2008). Compared with EL in the present study, similar changes in milk fat CLA have been reported in cows fed diets based on grass silage and corn silage (60:40, DM basis) supplemented with $2.8 \%$ of added oil in DMI from EL (Kliem et al., 2009). However, larger changes in CLA isomer concentrations have been reported for hay-based diet supplemented with $5.2 \%$ of linseed oil (Roy et al., 2006), than observed for EL in this experiment, probably due to both the amount and form of linseed lipid in the diet.

Effects of Rapeseed Form (ER vs. FRM vs. WR) During yr 1. Changes in milk CLA to FRM were shown to be of greater magnitude compared with previous investigations in cows fed diets based on corn silage and grass silage (75:25, DM basis) supplemented with $2.1 \%$ of added rapeseed oil in DMI (Hristov et al., 2011). Similarly, inclusion of $1.3 \%$ of rapeseed oil to red clover silage-based diets (Halmemies-Beauchet-Filleau et al., 2011) were shown to induce relatively minor changes in milk CLA and CLnA isomer concentrations compared with the effects of either ER or FRM in the present study. Differences in the magnitude of the responses between the present and earlier studies are probably related to both the oil level and the processing of rapeseeds. Treatment WR $(3.2 \%$ of added oil in DMI) induced only slight increases in milk trans11,cis-13 CLA and cis-9,trans-11,cis-15 CLnA, which is in line with the lack of changes in total milk trans FA concentration (Lerch et al., 2012b), and consistent with the absence of an increase in milk CLA concentrations to WR supplying $4.9 \%$ of added oil in DMI (Givens et al., 2009).

Interactions Between Treatment and Year. Significant interactions between treatment and year were observed for milk CLA and CLnA isomers abundance during indoor periods (especially for EL; Figure 5). Marginal increases in the amount of oil supplied from EL and ER between the first and the second indoor periods $(+0.3 \%$ and $+0.7 \%$ of added oil in DMI, re- spectively) do not, in isolation, explain these findings. Milk trans-11,cis-13 CLA, and cis-9,trans-11,cis-15 CLnA concentrations decreased from the first to the second indoor period, whereas enrichment of trans9,cis-11 CLA, trans-10,cis-12 CLA, nonconjugated trans 18:2, and trans 18:1 (including trans-6,-7,-8 and trans-10 18:1, Lerch et al., 2012b) increased (Figures 2 and 5). It is possible that such differences arose from the higher diet starch content for EL and ER during the second indoor period. A recent study demonstrated more extensive reduction of cis-9,trans-11,cis-15 CLnA and greater accumulation of trans-11,cis-15 18:2 and total trans 18:1 during incubations of 18:3n-3 with rumen fluid from cows fed diets containing higher amounts of starch compared with fluid from cows fed a control diet (34.8 vs. $21.5 \%$ starch in DMI; Zened et al., 2011).

Interactions between linseed supplementation and diet starch content on milk CLA concentration and distribution have been demonstrated in goats. Supplements of linseed oil in goats fed a corn silage-based diet resulted in higher enrichment of trans-10,cis-12 CLA, but lower cis-9,trans-11 CLA and trans-11,cis-13 CLA concentrations compared with adding the same amount of oil in a hay-based diet containing much lower levels of starch (6.9 vs. $22.0 \%$ in DMI, respectively; Bernard et al., 2009). Such an interaction may also explain why ER increased trans-11,cis-13 CLA in milk during the first indoor period $(2.9 \%$ of added oil and $5.5 \%$ of starch in DMI), whereas supplements of milled rapeseed or rapeseed oil in cows fed a corn silage-based diet (3.5 to $5.7 \%$ of added oil and $17 \%$ of starch in DMI) had no effect (Givens et al., 2009; Kliem et al., 2011). Oil by basal diet interactions may also account for a higher increase in milk cis-9,trans-11,cis-15 CLnA concentration to EL during the first indoor period $(3.2 \%$ of added oil and $5.4 \%$ of starch in DMI) compared with earlier reports of EL supplementation of a corn silage-based diet $(4.7 \%$ of added oil and starch content estimated to $22 \%$ in DMI; Akraim et al., 2007).

Alternatively, an increase in diet starch content may potentially alter RBH of 18:3n-3 that typically involves the formation of cis-9,trans-11,cis-15 CLnA, trans11,cis-15 18:2, and trans-11 18:1 as major intermediates (Palmquist et al., 2005) toward other metabolic pathways involving the formation and accumulation of trans-10,cis-12,cis-15 CLnA, trans-10,cis-15 18:2, and trans-10 18:1 in the rumen (Griinari and Bauman, 1999). However, detailed analysis of milk fat by GC-MS in the present study offered no evidence for the occurrence of 18:3 isomers containing a trans-10 double bond (results not shown). Furthermore, the increase in milk trans-11,cis-15 18:2 concentration due to EL was higher during the second than the first indoor period (Lerch et 
al., 2012b), which would tend to suggest that putative shifts in 18:3n-3 RBH pathways did not occur.

\section{Effects of Oilseed Supplements During Outdoor Periods}

Few interactions between treatment and year for the abundance of CLA and CLnA isomers were detected at pasture (Table 2 and Figure 5B). Changes in the overall milk FA profile to treatments were found to be more persistent during outdoor than indoor feeding periods over 2 successive lactations (Lerch et al., 2012b), which may be related to the almost identical composition of the concentrate mixtures and grazing management between the 2 outdoor periods (Figure 2). However, the decrease in milk cis-9,trans-11 CLA concentration due to oilseed supplements at pasture was unexpected, based on previous studies (Chilliard et al., 2002; Fearon et al., 2004). Analysis of all milk samples from the present study revealed that compared with the CTL, EL did not alter, and ER decreased, trans-11 18:1 concentration, whereas both supplements decreased the abundance of unresolved cis-9,trans-11 CLA, trans-7,cis-9 CLA, and trans-8,cis-10 CLA during outdoor periods (Lerch et al., 2012b). Detailed analysis confirmed that these changes were due to decreases in cis-9,trans-11 CLA in response to EL and ER supplements. Moreover, EL and ER also decreased milk trans-9,cis-11 CLA and trans-10,cis-12 CLA concentrations (Figure 2). These rather unexpected findings may be related to the CTL diet being relatively rich in $18: 2 n-6$ and $18: 3 n-3$ and containing more starch than either the EL or ER diets (Figure 2). Diets rich in PUFA and starch are known to stimulate the accumulation of trans-9,cis-11 CLA, trans-10,cis-12 CLA, and trans 18:1 isomers in the rumen, alterations in $\mathrm{RBH}$ that are often accompanied by a shift from trans-11 18:1 toward trans-10 18:1 as the major intermediate leaving the rumen (Griinari and Bauman, 1999; Palmquist et al., 2005). However, the CTL diet did not increase milk trans-10 18:1 concentration during outdoor periods, suggesting no substantial effects on RBH pathways (Lerch et al., 2012b). Nevertheless, it is likely that the higher starch content of the CTL relative to EL and ER increased the accumulation of trans-11 18:1, cis-9,trans-11 CLA, trans-9,cis-11 CLA, and trans-10,cis-12 CLA in the rumen.

In contrast to the reported responses to EL (465 g of added oil/d and $-1,355 \mathrm{~g}$ of starch/d compared with the CTL) and ER (465 $\mathrm{g}$ of added oil/d and $-1,285$ $\mathrm{g}$ of starch/d compared with the CTL) in the present study, previous studies demonstrated that rapeseed oil (500 $\mathrm{g}$ of added oil/d and $-290 \mathrm{~g}$ of starch/d compared with the control treatment) had no effect on milk cis9,trans-11 CLA, whereas linseed oil (500 g of added oil/d and $-395 \mathrm{~g}$ of starch/d compared with the control treatment) enriched this isomer in milk of grazing cows receiving $5 \mathrm{~kg}$ of concentrate/d (Rego et al., 2009). Under these conditions, milk trans-10, cis-12 CLA concentrations were not altered by rapeseed or linseed oil supplements (Rego et al., 2009). Such differences in the CLA isomer responses to lipid supplements are consistent with dietary starch substitution rates being important, although effects due to differences between oil and oilseeds cannot be excluded.

\section{Relationships Between Milk Fat Content or Yield, and trans $F A$}

Strong inverse associations were observed between within-period milk fat content or yield, milk trans-10 18:1, trans-9,cis-11 CLA, and trans-10,cis-12 CLA concentration responses to dietary treatments (Figure 6). These observations are in line with trans-10 18:1, trans9,cis-11 CLA, and trans-10,cis-12 CLA contributing to lowered milk fat secretion in lactating cows (Harvatine et al., 2009; Shingfield et al., 2010). However, the associations in the present experiment were linear, whereas curvilinear relationships have described variations in milk fat content or yield with concentrations of specific FA in milk in earlier investigations (Loor et al., 2005; Roy et al., 2006; Shingfield et al., 2010). Such differences can be explained by the much smaller changes in milk fat content across treatments in this study compared with earlier reports on milk fat composition and diet-induced milk fat depression.

In the present study, variations in milk fat content or yield were more closely associated with changes in milk fat trans-9,cis-11 CLA concentration compared with that between milk fat content or yield and trans-10 18:1 or trans-10,cis-12 CLA. In previous studies, increases in milk trans-10 18:1 concentration have been the most consistent and most closely correlated with decreases in milk fat content or secretion (Roy et al., 2006; Shingfield et al., 2006; 2010). Part of this discrepancy may be related to diets fed in the present study containing higher amounts of 18:3n-3 than 18:2n-6, whereas 18:2n6 predominated in corn silage-based diets containing sunflower oil (Roy et al., 2006; Shingfield et al., 2006).

In support of this, recent studies in vitro have reported that trans-10 18:1 and trans-10,cis-12 CLA accumulate to a greater extent during incubations of 18:2n-6 than 18:3n-3 with ruminal fluid (Zened et al., 2011). Furthermore, RBH of 18:3n-3 was recently reported to yield trans-9,cis-11 CLA as an intermediate (Lee and Jenkins, 2011), whereas incubations of 18:2n6 with rumen fluid have in some (Wallace et al., 2007), but not in all cases (Honkanen et al., 2012), resulted in the formation and accumulation of trans-9,cis-11 CLA. 


\section{CONCLUSIONS}

Dietary oilseed supplements altered the relative abundance and distribution of CLA and CLnA isomers in milk fat, with the changes being similar over 2 grazing seasons. Over 2 consecutive lactations, EL resulted in $\Delta 11,13$ CLA, $\Delta 12,14$ CLA, trans-9,trans-11 CLA, trans-13,trans-15 CLA, $\Delta 9,11,15$ CLnA, and cis9,trans-11,trans-13 CLnA enrichments. Supplements of $\mathrm{ER}$, and FRM in particular, led to a persistent increase in milk trans-7,cis-9 CLA concentration, whereas WR generally lowered milk cis-9,trans-11 CLA concentration. Significant interactions between the composition of the basal diet (conserved grass vs. pasture, diet starch content) and oilseed supplementation were observed. Compared with the CTL, EL and ER decreased milk cis-9,trans-11 CLA, trans-9, cis-11 CLA, and trans10, cis-12 CLA concentrations at pasture, in contrast to the changes during the indoor feeding periods. A higher starch content of diets during the second than the first indoor period was accompanied by a larger increase in trans-7,cis-9 CLA content, and lower enrichment of cis9,trans-11 CLA, cis-12,trans-14 CLA, trans-11,cis-13 CLA, and cis-9,trans-11,cis-15 CLnA in milk of cows fed EL and ER. Furthermore, EL increased milk trans9,cis-11 CLA and trans-10,cis-12 CLA concentrations during the second, but not in the first indoor period. Changes in specific nonconjugated 18:2, CLA, and CLnA isomers suggest that cis-9,trans-11,trans-13 CLnA and cis-9,trans-11,trans-15 CLnA may be synthesized endogenously in the mammary gland, whereas cis-9,trans-11,cis-15 CLnA in milk appears to originate from the rumen. Over the 2-yr experiment, changes in milk fat content or yield were inversely associated with milk trans-10 18:1, trans-10,cis-12 CLA, and trans-9,cis-11 CLA concentrations, the latter being the closest relationship, possibly due to the relatively high amounts of 18:3n-3 in experimental diets.

\section{ACKNOWLEDGMENTS}

The authors thank D. Pomiès, M. Barbet, and the team of the Monts d'Auvergne experimental unit of Orcival (INRA, UE1296, Orcival, France) for the feeding, milking, and diligent management of experimental cows, P. Capitan, C. Delavaud, E. Tixier, and D. Bany (INRA, UR1213, Saint-Genès Champanelle, France), as well as L. Ventto, A. Honkanen, and P. Kairenius (MTT, Animal Production Research, Jokioinen, Finland) for technical support during the analysis of milk FA composition, and B. Martin (INRA, UR1213) for helpful discussions. This research was supported, in part, by the European TRUEFOOD program (Traditional United Europe Food), an integrated project funded by the European Commission under the 6th Framework Program for RTD (Contract No. FOODCT-2006-016264). S. Lerch acknowledges and greatly appreciates the financial support of a $\mathrm{PhD}$ studentship provided by InVivo NSA (Paris, France), Organisation Nationale Interprofessionnelle des Graines et Fruits Oléagineux (ONIDOL, Paris, France), and Association Nationale de la Recherche Technique (ANRT, Paris, France), along with a Convention Industrielle de Formation à la Recherche en Entreprise (CIFRE) fellowship and funding awarded by the Centre for International Mobility (CIMO, Finland) to support an internship at MTT, Animal Production Research, Finland.

\section{REFERENCES}

Akraim, F., M. C. Nicot, R. Juaneda, and F. Enjalbert. 2007. Conjugated linolenic acid (CLnA), conjugated linoleic acid (CLA) and other biohydrogenation intermediates in plasma and milk fat of cows fed raw or extruded linseed. Animal 1:835-843.

AOAC International. 1997. Official Methods of Analysis. 16th ed AOAC Int., Gaithersburg, MD.

Bassaganya-Riera, J., A. J. Guri, and R. Hontecillas. 2011. Treatment of obesity-related complications with novel classes of naturally occurring PPAR agonists. J. Obes. 2011:897894. http://dx.doi. org $/ 10.1155 / 2011 / 897894$.

Bernard, L., K. J. Shingfield, J. Rouel, A. Ferlay, and Y. Chilliard. 2009. Effect of plant oils in the diet on performance and milk fatty acid composition in goats fed diets based on grass hay or maize silage. Br. J. Nutr. 101:213-224.

Chilliard, Y., A. Ferlay, J. Loor, J. Rouel, and B. Martin. 2002. Trans and conjugated fatty acids in milk from cows and goats consuming pasture or receiving vegetable oils or seeds. Ital. J. Anim. Sci. $1: 243-254$.

Chilliard, Y., F. Glasser, A. Ferlay, L. Bernard, J. Rouel, and M. Doreau. 2007. Diet, rumen biohydrogenation and nutritional quality of cow and goat milk fat. Eur. J. Lipid Sci. Technol. 109:828855.

Collomb, M., A. Schmid, R. Sieber, D. Wechsler, and E.-L. Ryhänen. 2006. Conjugated linoleic acids in milk fat: Variation and physiological effects. Int. Dairy J. 16:1347-1361.

Collomb, M., R. Sieber, and U. Bütikofer. 2004. CLA isomers in milk fat from cows fed diets with high levels of unsaturated fatty acids. Lipids 39:355-364.

Corl, B. A., L. H. Baumgard, J. M. Griinari, P. Delmonte, K. M. Morehouse, M. P. Yurawecz, and D. E. Bauman. 2002. Trans7,cis-9 CLA is synthesized endogenously by delta-9-desaturase in dairy cows. Lipids 37:681-688.

Couvreur, S., C. Hurtaud, C. Lopez, L. Delaby, and J. L. Peyraud. 2006. The linear relationship between the proportion of fresh grass in the cow diet, milk fatty acid composition, and butter properties. J. Dairy Sci. 89:1956-1969.

Faverdin, P., R. Delagarde, L. Delaby, and F. Meschy. 2007. Almentation des vaches laitières. Pages 23-58 in Alimentation des bovins, ovins et caprins. Besoins des Animaux - Valeur des aliments-Table INRA 2007. E. Quae, ed. INRA, Versailles, France.

Fearon, A. M., C. S. Mayne, J. A. M. Beattie, and D. W. Bruce. 2004 Effect of level of oil inclusion in the diet of dairy cows at pasture on animal performance and milk composition and properties. J. Sci. Food Agric. 84:497-504.

Givens, D. I., K. E. Kliem, D. J. Humphries, K. J. Shingfield, and R. Morgan. 2009. Effect of replacing calcium salts of palm oil distillate with rapeseed oil, milled or whole rapeseeds on milk fattyacid composition in cows fed maize silage-based diets. Animal 3:1067-1074.

Gómez-Cortés, P., C. Tyburczy, J. T. Brenna, M. Juárez, and M. A. de la Fuente. 2009. Characterization of cis-9 trans-11 trans-15 C18:3 
in milk fat by GC and covalent adduct chemical ionization tandem MS. J. Lipid Res. 50:2412-2420.

Griinari, J. M., and D. E. Bauman. 1999. Biosynthesis of conjugated linoleic acid and its incorporation into meat and milk in ruminants. Advances in conjugated linoleic acid research, volume 1. AOCS Press, Champaign, IL.

Griinari, J. M., B. A. Corl, S. H. Lacy, P. Y. Chouinard, K. V. V. Nurmela, and D. E. Bauman. 2000. Conjugated linoleic acid is synthesized endogenously in lactating dairy cows by $\Delta^{9}$-desaturase. J. Nutr. 130:2285-2291.

Halmemies-Beauchet-Filleau, A., T. Kokkonen, A.-M. Lampi, V. Toivonen, K. J. Shingfield, and A. Vanhatalo. 2011. Effect of plant oils and camelina expeller on milk fatty acid composition in lactating cows fed diets based on red clover silage. J. Dairy Sci. 94:4413-4430.

Harvatine, K. J., Y. R. Boisclair, and D. E. Bauman. 2009. Recent advances in the regulation of milk fat synthesis. Animal 3:40-54.

Hennessy, A. A., R. P. Ross, R. Devery, and C. Stanton. 2011. The health promoting properties of the conjugated isomers of alphalinolenic acid. Lipids 46:105-119.

Honkanen, A. M., J. M. Griinari, A. Vanhatalo, S. Ahvenjärvi, V. Toivonen, and K. J. Shingfield. 2012. Characterization of the disappearance and formation of biohydrogenation intermediates during incubations of linoleic acid with rumen fluid in vitro. J. Dairy Sci. 95:1376-1394.

Hopkins, C. Y., and M. J. Chisholm. 1968. A survey of the conjugated fatty acids of seed oils. J. Am. Oil Chem. Soc. 45:176-182.

Hristov, A. N., C. Domitrovich, A. Wachter, T. Cassidy, C. Lee, K. J. Shingfield, P. Kairenius, J. Davis, and J. Brown. 2011. Effect of replacing solvent-extracted canola meal with high-oil traditional canola, high-oleic acid canola, or high-erucic acid rapeseed meals on rumen fermentation, digestibility, milk production, and milk fatty acid composition in lactating dairy cows. J. Dairy Sci. 94:4057-4074.

Kliem, K. E., P. C. Aikman, D. J. Humphries, R. Morgan, K. J. Shingfield, and D. I. Givens. 2009. Effect of replacing calcium salts of palm oil distillate with extruded linseeds on milk fatty acid composition in Jersey and Holstein cows. Animal 3:1754-1762.

Kliem, K. E., K. J. Shingfield, D. J. Humphries, and D. I. Givens. 2011. Effect of replacing calcium salts of palm oil distillate with incremental amounts of conventional or high oleic acid milled rapeseed on milk fatty acid composition in cows fed maize silage-based diets. Animal 5:1311-1321.

Koba, K., M. A. Belury, and M. Sugano. 2007. Potential health benefits of conjugated trienoic acids. Lipid Technol. 19:200-203.

Kraft, J., M. Collomb, P. Möckel, R. Sieber, and G. Jahreis. 2003. Differences in CLA isomer distribution of cow's milk lipids. Lipids 38:657-664.

Lee, Y.-J., and T. C. Jenkins. 2011. Biohydrogenation of linolenic acid to stearic acid by the rumen microbial population yields multiple intermediate conjugated diene isomers. J. Nutr. 141:1445-1450.

Lerch, S., A. Ferlay, D. Pomiès, B. Martin, J. A. A. Pires, and Y. Chilliard. 2012a. Rapeseed or linseed supplements in grass-based diets: Effects on dairy performance of Holstein cows over two consecutive lactations. J. Dairy Sci. 95:1956-1970.

Lerch, S., A. Ferlay, K. J. Shingfield, B. Martin, D. Pomiès, and Y. Chilliard. 2012b. Rapeseed or linseed supplements in grass-based diets: Effects on milk fatty acid composition of Holstein cows over two consecutive lactations. J. Dairy Sci. 95:5221-5241.

Loor, J. J., A. Ferlay, A. Ollier, and Y. Chilliard. 2005. Relationship among trans and conjugated fatty acids and bovine milk fat yield due to dietary concentrate and linseed oil. J. Dairy Sci. 88:726-740.

Mohammed, R., C. S. Stanton, J. J. Kennelly, J. K. G. Kramer, J. F. Mee, D. R. Glimm, M. O'Donovan, and J. J. Murphy. 2009. Grazing cows are more efficient than zero-grazed and grass silage-fed cows in milk rumenic acid production. J. Dairy Sci. 92:3874-3893.

Morand-Fehr, P., and G. Tran. 2001. La fraction lipidique des aliments et les corps gras utilisés en alimentation animale. INRA Prod. Anim. 14:285-302.
Palmquist, D. L., A. L. Lock, K. J. Shingfield, and D. E. Bauman. 2005. Biosynthesis of conjugated linoleic acid in ruminants and humans. Adv. Food Nutr. Res. 50:179-217.

Plourde, M., F. Destaillats, P. Y. Chouinard, and P. Angers. 2007. Conjugated $\alpha$-linolenic acid isomers in bovine milk and muscle. J. Dairy Sci. 90:5269-5275.

Pollard, M. R., F. D. Gunstone, A. T. James, and L. J. Morris. 1980. Desaturation of positional and geometric isomers of monoenoic fatty acids by microsomal preparations from rat liver. Lipids 15:306-314.

Rego, O. A., S. P. Alves, L. M. S. Antunes, H. J. D. Rosa, C. F. M. Alfaia, J. A. M. Prates, A. R. J. Cabrita, A. J. M. Fonseca, and R. J. B. Bessa. 2009. Rumen biohydrogenation-derived fatty acids in milk fat from grazing dairy cows supplemented with rapeseed, sunflower, or linseed oils. J. Dairy Sci. 92:4530-4540.

Roy, A., A. Ferlay, K. J. Shingfield, and Y. Chilliard. 2006. Examination of the persistency of milk fatty acid composition responses to plant oils in cows given different basal diets, with particular emphasis on trans- $\mathrm{C}_{18: 1}$ fatty acids and isomers of conjugated linoleic acid. Anim. Sci. 82:479-492.

Sæbø, A., P.-C. Sæbø, J. M. Griinari, and K. J. Shingfield. 2005. Effect of abomasal infusions of geometric isomers of 10,12 conjugated synthesis linoleic acid on milk fat in dairy cows. Lipids 40:823-832.

Sauvant, D., J.-M. Perez, and G. Tran. 2004. Tables de composition et de valeur nutritive des matières premières destinées aux animaux d'élevage: Porcs, volailles, bovins, ovins, caprins, lapins, chevaux, poissons, 2ème édition revue et corrigée. INRA Editions, Versailles, France.

Shingfield, K. J., S. Ahvenjärvi, V. Toivonen, A. Ärölä, K. V. V. Nurmela, P. Huhtanen, and J. M. Griinari. 2003. Effect of dietary fish oil on biohydrogenation of fatty acids and milk fatty acid content in cows. Anim. Sci. 77:165-179.

Shingfield, K. J., L. Bernard, C. Leroux, and Y. Chilliard. 2010. Role of trans fatty acids in the nutritional regulation of mammary lipogenesis in ruminants. Animal 4:1140-1166.

Shingfield, K. J., Y. Chilliard, V. Toivonen, P. Kairenius, and D. I. Givens. 2008. Trans fatty acids and bioactive lipids in ruminant milk. Pages 3-65 in Bioactive Components of Milk. Vol. 606. Springer-Verlag Berlin, Germany.

Shingfield, K. J., C. K. Reynolds, G. Hervás, J. M. Griinari, A. S. Grandison, and D. E. Beever. 2006. Examination of the persistency of milk fatty acid composition responses to fish oil and sunflower oil in the diet of dairy cows. J. Dairy Sci. 89:714-732.

Shingfield, K. J., C. K. Reynolds, B. Lupoli, V. Toivonen, M. P. Yurawecz, P. Delmonte, J. M. Griinari, A. S. Grandison, and D. E. Beever. 2005. Effect of forage type and proportion of concentrate in the diet on milk fatty acid composition in cows given sunflower oil and fish oil. Anim. Sci. 80:225-238.

Spitzer, V., F. Marx, and K. Pfeilsticker. 1994. Electron impact mass spectra of the oxazoline derivatives of some conjugated diene and triene $\mathrm{C}_{18}$ fatty acids. J. Am. Oil Chem. Soc. 71:873-876.

Wahle, K. W. J., S. D. Heys, and D. Rotondo. 2004. Conjugated linoleic acids: Are they beneficial or detrimental to health? Prog. Lipid Res. 43:553-587.

Wallace, R. J., N. McKain, K. J. Shingfield, and E. Devillard. 2007. Isomers of conjugated linoleic acids are synthesized via different mechanisms in ruminal digesta and bacteria. J. Lipid Res. 48:2247-2254.

Wąsowska, I., M. R. G. Maia, K. M. Niedźwiedzka, M. Czauderna, J. M. C. Ramalho Ribeiro, E. Devillard, K. J. Shingfield, and R. J. Wallace. 2006. Influence of fish oil on ruminal biohydrogenation of C18 unsaturated fatty acids. Br. J. Nutr. 95:1199-1211.

Zened, A., A. Troegeler-Meynadier, M. C. Nicot, S. Combos, L. Cauquil, Y. Farizon, and F. Enjalbert. 2011. Starch and oil in the donor cow diet and starch in substrate differently affect the in vitro ruminal biohydrogenation of linoleic and linolenic acids. J. Dairy Sci. 94:5634-5645. 